\title{
Declarative approach to cyclic steady state space refinement: periodic process scheduling
}

\author{
Grzegorz Bocewicz • Zbigniew A. Banaszak
}

Received: 16 April 2012 / Accepted: 13 October 2012 / Published online: 7 February 2013

(C) The Author(s) 2013. This article is published with open access at Springerlink.com

\begin{abstract}
Problems of cyclic scheduling are usually observed in flexible manufacturing systems which produce multitype parts where the automated guided vehicle system plays the role of a material handling system, as well as in various other multimodal transportation systems where goods and/or passenger itinerary planning plays a pivotal role. The schedulability analysis of the processes executed in the so-called systems of concurrent cyclic processes (SCCPs) can be executed within a declarative modeling framework. Consequently, the considered SCCP scheduling problem can be seen as a constraint satisfaction problem. Such a representation provides a unified way for evaluating the performance of local cyclic processes as well as of multimodal processes supported by them. Here, the crucial issue is that of a control procedure (e.g., a set of dispatching rules), which would guarantee the cyclic behavior of the SCCP. In this context, we discuss the sufficient conditions guaranteeing the schedulability of both local and multimodal cyclic processes, and we propose a recursive approach in designing them.
\end{abstract}

Keywords Cyclic processes - Declarative modeling . Constraint programming $\cdot$ State space $\cdot$ Periodicity . Dispatching rules

\section{Introduction}

Operations in cyclic processes are executed in sequences that repeat an indefinite number of times. In everyday

G. Bocewicz $(\bowtie)$

Department of Electronics and Computer Science, Koszalin University of Technology, Koszalin, Poland e-mail: bocewicz@ie.tu.koszalin.pl

\section{Z. A. Banaszak}

Department of Business Informatics,

Warsaw University of Technology, Warsaw, Poland

e-mail: Z.Banaszak@wz.pw.edu.pl practice, these processes arise in different application domains (such as manufacturing, time sharing of processors in embedded systems, digital signal processing, and in compilers where scheduling loop operations for parallel or pipelined architectures takes place) as well as in service domains, covering such areas as workforce scheduling (e.g., shift scheduling, crew scheduling), timetabling (e.g., train timetabling, aircraft routing and scheduling), and reservations (e.g., reservations with or without slack, assigning classes to rooms) $[7,10,11,14,15,19,21,23,24]$. Such cyclic scheduling problems belong to the class of decision problems, i.e., problems aimed at finding whether or not there exists a solution satisfying certain assumed conditions [21]. Moreover, because of their integer domains, the considered problems belong to a class of Diophantine problems $[9,18]$. This means that certain classes of cyclic scheduling problems can be seen as undecidable [1].

Therefore, taking into account the undecidability of Diophantine problems, not all the behaviors (including cyclic behaviors, i.e., those that belong to a space of cyclic steady states) are reachable under the constraints imposed by the structure of the system. This is also the case with the system behavior that can be achieved in systems possessing specific structural constraints. This means that since the system's constraints determine its behavior, both the system structure configuration and the desired cyclic schedule must be considered simultaneously. Thus, for the solution of a cyclic scheduling problem, the configuration of a system structure must be determined in order to enable scheduling of the processes; however, the scheduling must be performed so as to devise the system configuration.

In this context, this work discusses certain solvability issues concerning the problems of dispatching cyclic processes, in particular the conditions guaranteeing the solvability of the scheduling of cyclic processes. Their examination may replace exhaustive and time-consuming searching for the solutions satisfying the required system functioning. 
A number of models and methods have been proposed to solve the cyclic scheduling problem [13]. Among them, the most frequently applied are the mathematical programming approaches (usually integer programming or mixed integer programming [24]), max-plus algebra [16], constraint logic programming $[2-5,22]$, swarm and evolutionary algorithms $[6,10,20]$, and Petri nets [19]. The majority of these are oriented at finding a minimum cycle or maximum throughput while assuming a deadlock-free flow of processes. The approaches aimed at estimating the cycle time based on the cyclic process structure and the employed synchronization mechanism (i.e., rendezvous or mutual exclusion instances) while taking into account deadlock avoidance constraints are rather unique $[8,22,25]$.

In this context, apart from the above-mentioned solvability conditions concerning priority dispatching rules guaranteeing the reachability of a cyclic steady state of a system, our main contribution consists in proposing a new modeling framework for evaluating the cyclic steady state of a given system of concurrent cyclic processes (SCCP). This paper is concerned with the following questions: can the assumed system behavior be achieved under the given constraints on the structure of the system? Can we find such a system structure for which the assumed system behavior can be achieved?

Therefore, the aim of this paper is to provide the conditions useful for generating cyclic steady states in a system consisting of concurrently interacting cyclic processes, where mutual exclusion protocols provide a synchronization mechanism which controls the access of the processes to shared (common) resources. This objective encompasses a rather large class of digital and/or logistic networks that have common properties, even though their intrinsic differences are significant. The most important property is related to different subnetwork infrastructures which enable scheduling multimodal processes executed through common shared sections of different local networks [3]. For instance, in the case of a metro network, the itinerary of a given passenger, assuming line changes, is an example of a multimodal process, where itinerary planning can be viewed as a relevant multimodal process scheduling.

Consequently, this study aims to present a declarative approach to the reachability problem that can be employed by decision makers in order to generate, analyze, and evaluate cyclic steady states reachable in a given SCCP structure. By employing the framework of the constraint satisfaction problem (CSP) [5], we state our main problem regarding the dispatching rules resulting in the SCCP cyclic schedules of the assumed cycle time and the $\Xi$-periodicity of local processes. An illustrative model of the constraint satisfaction problem implemented in the Oz/Mozart language is discussed from the perspective of multimodal processes.
This contribution can be seen as a continuation of our former work [3-5]. Hence, our approach can be viewed as an extension of the concept of constraints sufficient for the cyclic behavior of local and multimodal processes [5], as well as an extension of the concept of the state space introduced in $[3,4]$. This means that the conditions introduced earlier which guarantee the cyclic behavior of an SCCP and the generation of its state space provide a formal framework for the development of reachability conditions guaranteeing the generation of cyclic steady states, i.e., the conditions which allow to distinguish between the initial states and the cyclic steady states in both local and multimodal processes.

The rest of the paper is organized as follows: Section 2 introduces systems of concurrently flowing local cyclic and multimodal processes, describes the employed notation, and states the problem. In Section 3, we discuss certain issues concerning the generation of feasible state space as well as provide two methods aimed at refining cyclic steady states. Two cases illustrating the implementation of both methods are discussed in Section 4, whereas Section 5 presents our conclusions.

\section{Systems of concurrent cyclic processes}

\subsection{Declarative modeling}

An automated guided vehicle system (AGVS) with distinguishable vehicles, pick-up/delivery points (PDPs), and transportation routes is presented in Fig. 1. Such a system can be modeled in terms of SCCPs, where cyclic multimodal processes (representing transportation routes) are executed along the parts of cyclic local processes (represented by the itineraries of vehicles) which are interconnected with each other through common resources of the AGVS (i.e., PDPs). Figure 2 presents the SCCP model from Fig. 1.

Six local cyclic processes are considered, viz., $P_{1}, P_{2}, P_{3}$, $P_{4}, P_{5}$, and $P_{6}$. The processes follow the routes composed of transportation sectors (distinguished in Fig. 2 by the set of resources $R=\left\{R_{1}, \ldots, R_{c}, \ldots, R_{17}\right\}$, where $R_{c}$ is the $c$ th resource). Some of the local cyclic processes are pipeline flow processes, i.e., they contain streams (representing vehicles from Fig. 1) of the processes following the same route while occupying different resources (sectors). For instance, processes $P_{4}$ and $P_{5}$ contain two streams: $P_{4}=$ $\left\{P_{4}^{1}, P_{4}^{2}\right\}$, and $P_{5}=\left\{P_{5}^{1}, P_{5}^{2}\right\}$, respectively, i.e., the processes (vehicles) moving along the same route. The remaining local processes contain unique streams: $P_{1}=\left\{P_{1}^{1}\right\}, P_{2}=\left\{P_{2}^{1}\right\}$, $P_{3}=\left\{P_{3}^{1}\right\}$, and $P_{6}=\left\{P_{6}^{1}\right\}$. In other words, the streams $P_{1}^{1}, P_{2}^{1}, P_{3}^{1}, P_{4}^{1}, P_{4}^{2}, P_{5}^{1}, P_{5}^{2}$, and $P_{6}^{1}$ represent the eight vehicles from Fig. 1 . The $k$ th stream of the $i$ th local process $P_{i}$ is denoted as $P_{i}^{k}$. 
Fig. 1 Example of an AGVS

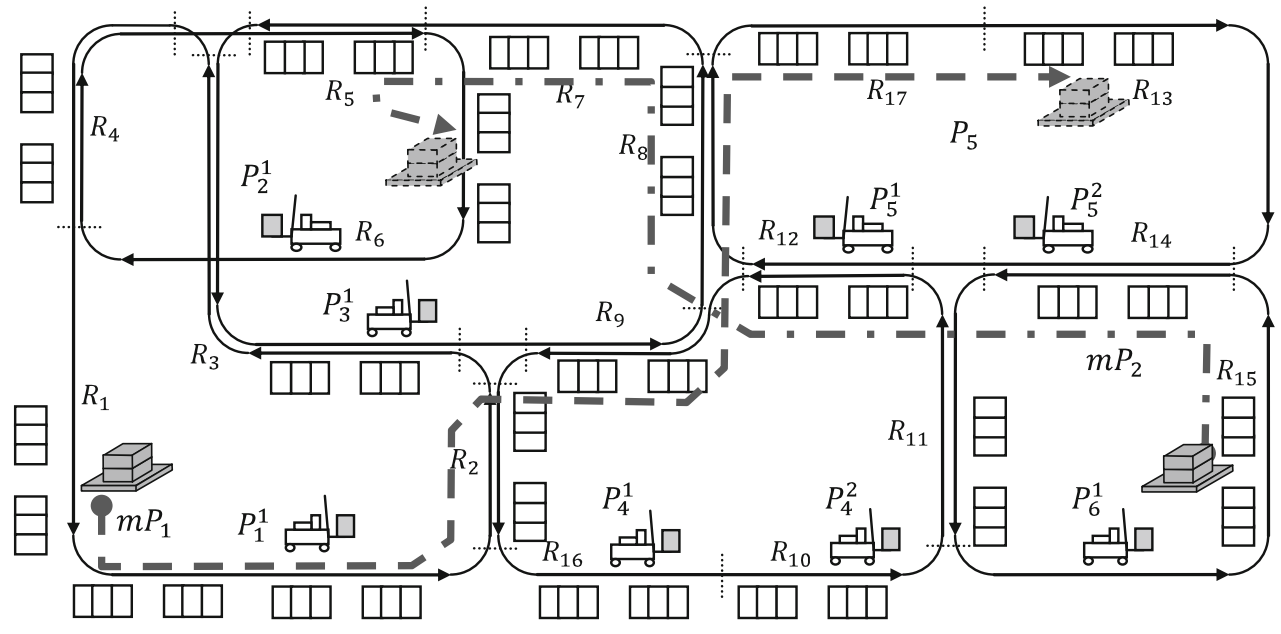

Legend:

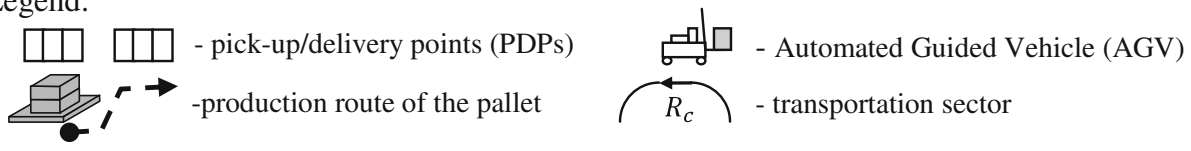

Apart from local processes, we consider two multimodal processes (i.e., processes executed along the routes consisting of parts of the routes of local processes): $m P_{1}$ and $m P_{2}$.

For example, the displacement of the pallet along the production route depicted by the dashed line corresponds to the multimodal process $m P_{1}$ supported by AGVs, which in turn encompass local transportation streams, $P_{1}^{1}$ and $P_{3}^{1}$, and then also one of two pipeline-like flowing streams: $P_{5}^{1}$ and $P_{5}^{2}$. This means that the production/transportation route specifying how a multimodal process is executed can be seen as composed of parts of the routes of local cyclic processes.
Processes can interact with each other through shared resources, i.e., the transportation sectors. The routes of the considered local processes (streams) are as follows:

$$
\begin{gathered}
p_{1}^{1}=\left(R_{1}, R_{2}, R_{3}, R_{4}\right), p_{2}^{1}=\left(R_{4}, R_{5}, R_{6}\right), \\
p_{3}^{1}=\left(R_{3}, R_{9}, R_{8}, R_{7}, R_{5}\right), p_{4}{ }^{1}=p_{4}{ }^{2}=\left(R_{2}, R_{16}, R_{10}, R_{11}, R_{12}, R_{9}\right), \\
p_{5}^{1}=p_{5}^{2}=\left(R_{8}, R_{17}, R_{13}, R_{14}, R_{12}\right), p_{6}^{1}=\left(R_{14}, R_{11}, R_{15}\right),
\end{gathered}
$$

where $R_{2}-R_{5}, R_{8}-R_{14}, R_{16}$, and $R_{17}$ are the shared resources, since each of them is used by at least two streams, and $R_{1}$, $R_{6}, R_{7}$, and $R_{15}$ are the non-shared resources, because they are exclusively used by one stream only. In general, the route $p_{i}^{k}$ is the sequence of resources used in order to execute the
Fig. 2 Example of FMS-SCCP model of an AGVS

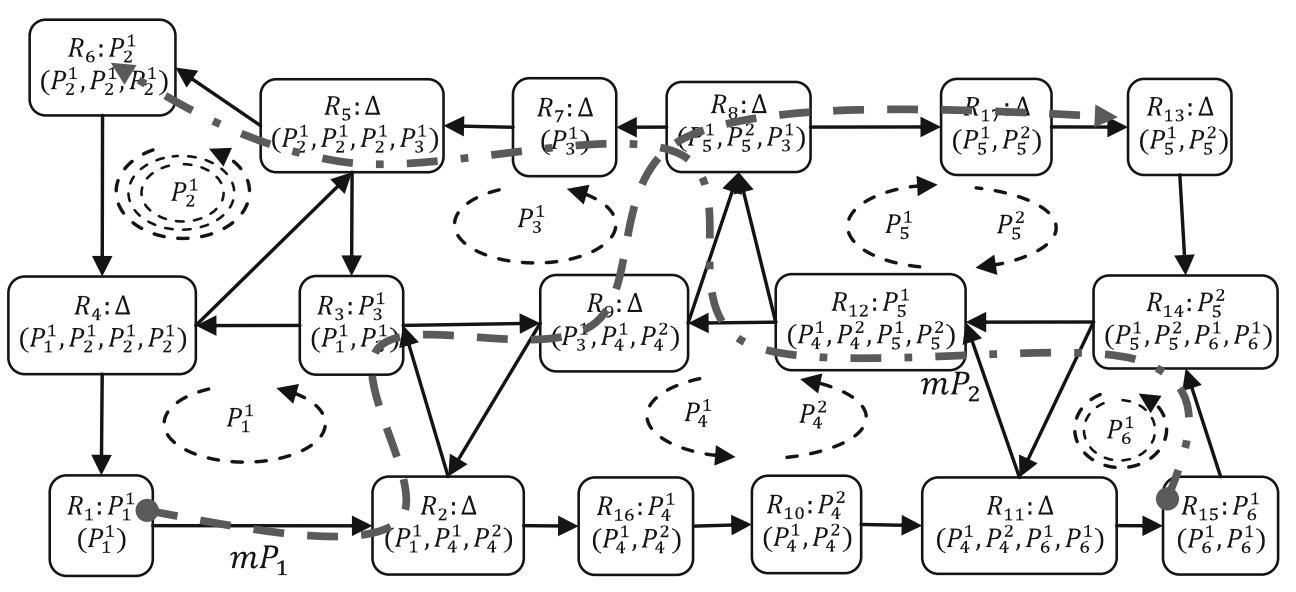

Legend:

$R_{c}-c$-th resource of set $R=\left\{R_{1}, \ldots, R_{c}, \ldots, R_{m}\right\}$
$\begin{gathered}R_{c}: P_{i}^{k} \\ s_{c, 1}, \ldots, s_{c, l p(c)}\end{gathered}$ $\begin{aligned} & \begin{array}{l}\text { resource } R_{c} \text { occupied by the stream } P_{i}^{k} \text { and controlled by the priority } \\ \text { dispatching rule } \sigma_{c}=\left(s_{c, 1}, \ldots, s_{c, d}, \ldots, s_{c, l p(c)}\right) ; s_{c, d} \in H(H \text { - a set of streams })\end{array} \\ & \text { - unoccupied resource: } R_{c}\end{aligned}$


operations of the stream $P_{i}^{k}$. Note that the streams $p_{4}^{1}$ and $p_{4}^{2}$ which belong to $P_{4}$ and the streams $p_{5}^{1}$ and $p_{5}^{2}$ which belong to $P_{5}$ follow the same route (these streams correspond to vehicles moving along the same route).

Consider two cyclic multimodal processes $m P_{1}$ and $m P_{2}$ which follow the routes $m p_{1}$ and $m p_{2}$, respectively (see Fig. 2):

$m p_{1}=\left(R_{1}, R_{2}, R_{3}, R_{9}, R_{8}, R_{17}, R_{13}\right)$,
$m p_{2}=\left(R_{15}, R_{14}, R_{12}, R_{8}, R_{7}, R_{5}, R_{6}\right)$.

Let us assume that $m p_{1}$ and $m p_{2}$ can be seen as follows:

$$
\begin{gathered}
m p_{1}=\left(\left(R_{1}, R_{2}, R_{3}\right),\left(R_{9}, R_{8}\right),\left(R_{17}, R_{13}\right)\right), \\
m p_{2}=\left(\left(R_{15}, R_{14}\right),\left(R_{12}, R_{8}\right),\left(R_{7}, R_{5}\right),\left(R_{6}\right)\right),
\end{gathered}
$$

where:

$\left(R_{1}, R_{2}, R_{3}\right),\left(R_{9}, R_{8}\right),\left(R_{17}, R_{13}\right)$ - the parts (subsequences) of routes $p_{1}^{1}, p_{3}^{1}$ and $p_{5}^{1}$ included in $m p_{1}$ $\left(R_{15}, R_{14}\right),\left(R_{12}, R_{8}\right),\left(R_{7}, R_{5}\right),\left(R_{6}\right)$ - the parts (subsequences) of routes $p_{6}^{1}, p_{5}^{1}, p_{3}^{1}$, and $p_{2}^{1}$ included in $m p_{2}$.

Let us assume that multimodal processes do not contain subprocesses, i.e., that each multimodal process consists of a unique stream.

The class of the considered SCCPs follows the constraints stated below [5]:

- A new operation may start on a resource only if the current operation has been completed and the resource has been released,

- Local processes share common resources in the mutual exclusion mode, the operation of a local process can only be suspended if the necessary resource is occupied, suspended local processes cannot be released, and local processes are non-preemptible, i.e., a resource may not be taken from a process as long as it is used by it,

- Multimodal processes encompassing pallet flow conveyed by AGVs follow local transportation routes, and different multimodal processes can be executed simultaneously along the same local process,

- Local and multimodal processes execute cyclically with periods $T c$ and $T m$, respectively; resources occur uniquely in each transportation route,

- In a cyclic steady state, each $i$ th stream must cover its local route the same number of times $\Xi \cdot \psi_{i}$; the factors $\Xi$ and $\psi_{i}$ are defined below.

A resource conflict (caused by the application of the mutual exclusion protocol) is resolved with the aid of a priority dispatching rule [2], which determines the order in which streams access shared resources. For instance, in the case of the resource $R_{9}, \sigma_{9}=\left(P_{3}^{1}, P_{4}^{1}, P_{4}^{2}\right)$, the priority dispatching rule determines the order in which streams can access the shared resource $R_{9}$, i.e., the resource is first given to stream $P_{3}^{1}$, then to the stream $P_{4}^{1}$, then to $P_{4}^{2}$ and then once again to $P_{3}^{1}$, and so on. The stream $P_{i}^{k}$ occurs the same number of times in each dispatching rule associated with the resources featuring in its route. Therefore, the SCCP shown in Fig. 2 is specified by the following set of dispatching rules: $\Theta=\left\{\sigma_{1}, \ldots, \sigma_{17}\right\}$, as well as $f_{1}\left(P_{1}^{1}\right)=f_{2}\left(P_{1}^{1}\right)=f_{3}$ $\left(P_{1}^{1}\right)=f_{4}\left(P_{1}^{1}\right)=1, f_{4}\left(P_{2}^{1}\right)=f_{5}\left(P_{2}^{1}\right)=f_{6}\left(P_{2}^{1}\right)=3$, etc., where $f_{c}\left(P_{i}^{k}\right)$ is the number of occurrences of $P_{i}^{k}$ in the $c$ th priority dispatching rule. This means that during the same period, the stream $P_{2}^{1}$ is repeated three times, while $P_{1}^{1}$ only once.

Thus, the priority rules determine the frequencies of the mutual appearance of local processes sharing the same resource. In general, the set of dispatching rules $\Theta$ determines the sequence of relative frequencies of mutual executions of local processes, and is denoted by $\Psi=\left(\psi_{1}, \psi_{2}, \ldots, \psi_{n}\right)$, where $\psi_{i} \in \mathbb{N}$,

$\psi_{i}=\left\|\left\{d \mid s_{c, d}=P_{i}^{1} ; d \in\{1, \ldots, l p(c)\}\right\}\right\|, \forall i \in\{1, \ldots, n\}, \forall \sigma_{c} \in \Theta_{i}$,

where $\Theta_{i}$ is the set of dispatching rules associated with the resources featuring in the route followed by $P_{i}, s_{c, d}$ is the $d$ th element in the sequence $\sigma_{c}=\left(s_{c, 1}, \ldots, s_{c, d}, \ldots, s_{c, l p(c)}\right), n$ is the number of processes, and $l p(c)$ is the length of $\sigma_{c}$.

Therefore, the SCCP shown in Fig. 2 is specified by the sequence: $\Psi=(1,3,1,1,1,2)$. This means that one execution of local processes $P_{1}, P_{3}, P_{4}$, and $P_{5}$ (and their associated streams) corresponds to three executions of process $P_{2}$ and two executions of $P_{6}$.

Since the sequence $\Psi$ of relative frequencies of mutual executions of local processes does not necessary encompass a cyclic steady state of the SCCP, we introduced a new parameter describing the number of occurrences of $\Psi$ in a cyclic steady state, denoted by $\Xi \in \mathbb{N}$. $\Xi$ occurrences of $\Psi$ in one period is termed $\Xi$-periodicity. For the considered SCCP, $\Xi=2$ implies that two executions of the sequence $\Psi=$ $(1,3,1,1,1,2)$, i.e., two executions of local processes $P_{1}, P_{3}$, $P_{4}$, and $P_{5}$ correspond to six executions of the process $P_{2}$ and four executions of $P_{6}$. We can define the mutual frequency $m \Psi$ of multimodal processes $m P_{i}$ (e.g., in the case of the execution of multimodal processes (see the SCCP in Fig. 2), $m \Psi=(1,1)$ implies that one execution of the process $m P_{1}$ corresponds to one execution of $m P_{2}$ ). We can similarly define $m \Xi$, which determines the number of executions of $m \Psi$ in a cycle.

In general, the following notation is used:

- A sequence $p_{i}^{k}=\left(p_{i, 1}^{k}, p_{i, 2}^{k}, \ldots, p_{i, j}^{k}, \ldots, p_{i, l r(i)}^{k}\right)$ specifies the route of the stream of a local process $P_{i}^{k}$ (the $k$ th stream of the $i$ th local process $P_{i}$ ). Its components define the resources used in the execution of operations, where 
$p_{i, j}^{k} \in R$ (the set of resources $R=\left\{R_{1}, R_{2}, \ldots, R_{\mathrm{c}}, \ldots, R_{m}\right\}$ ) denotes the resources used by the $k$ th stream of the $i$ th local process in the $j$ th operation. In the rest of the paper, the jth operation executed on the resource $p_{i, j}^{k}$ in the stream $P_{i}^{k}$ will be denoted by $o_{i, j}^{k} ; \operatorname{lr}(i)$ is the length of the cyclic process route (all streams of $P_{i}$ are of the same length). For example, the route $p_{2}^{1}=\left(R_{4}, R_{5}, R_{6}\right)$ of the stream $P_{2}^{1}$ (Fig. 2) is the sequence $p_{2}^{1}=\left(p_{1,1}^{1}, p_{1,2}^{1}, p_{1,3}^{1}\right)$, where the first element $p_{1,1}^{1}$ is equal to $R_{4}$, whereas the second, $p_{1,2}^{1}=R_{5}$, and the third, $p_{1,3}^{1}=R_{6}$.

- $\quad x_{i, j, q}^{k}(l) \in \mathbb{N}$ is the moment when the operation $o_{i, j}^{k}$ begins its $q$ th execution in the $l$ th cycle.

- $t_{i}^{k}=\left(t_{i, 1}^{k}, t_{i, 2}^{k}, \ldots, t_{i, j}^{k}, \ldots, t_{i, l r(i)}^{k}\right)$ specifies the operation times of local processes, where $t_{i, j}^{k}$ denotes the time of execution of operation $o_{i, j}^{k}$ (for the SCCP in Fig. 2, see Table 1).

- $\quad m p_{i}=\left(m p r_{i_{1}}^{k_{1}}\left(a_{i_{1}}, b_{i_{1}}\right), m p r_{i_{2}}^{k_{2}}\left(a_{i_{2}}, b_{i_{2}}\right), \ldots, m p r_{i_{y}}^{k_{y}}\left(a_{i_{y}}, b_{i_{y}}\right)\right)$ specifies the route of the multimodal process $m P_{i}$, where

$$
m p r_{i}^{k}(a, b)=\left\{\begin{array}{cc}
\left(p_{i, a}^{k}, p_{i, a+1}^{k}, \ldots, p_{i, b}^{k}\right) & a \leq b \\
\left(p_{i, a}^{k}, p_{i, a+1}^{k} \ldots, p_{i, l r(i)}^{k}, p_{i, 1}^{k}, \ldots, p_{i, b-1}^{k}, p_{i, b}^{k}\right) & b>a \\
a, b \in\{1, \ldots, \operatorname{lr}(i)\},
\end{array},\right.
$$

is the subsequence of the route $p_{i}^{k}=\left(p_{i, 1}^{k}, \quad p_{i, 2}^{k}, \ldots\right.$, $\left.p_{i, j}^{k}, \ldots, p_{i, l r(i)}^{k}\right)$ containing elements from $p_{i, a}^{k}$ to $p_{i, b}^{k}$.

In other words, the transportation route $m p_{i}$ is a sequence of parts of routes of local processes. For instance, the route followed by $m P_{1}$ (see Fig. 2) is as follows: $m p_{1}=\left(\left(R_{1}, R_{2}, R_{3}\right),\left(R_{9}, R_{8}\right),\left(R_{17}, R_{13}\right)\right)$, where $m p r_{1}^{1}(1,3)=\left(R_{1}, R_{2}, R_{3}\right), \operatorname{mpr}_{3}^{1}(2,3)=\left(R_{9}, R_{8}\right)$, and $m$ $p r_{5}^{1}(2,3)=\left(R_{17}, R_{13}\right)$.

In the rest of the paper, the jth operation executed in the process $m P_{i}$ will be denoted by $m o_{i, j}$.

- $m x_{i, j, k}(l) \in \mathbb{N}$ is the moment when the operation $m o_{i, j}$ begins its $k$ th execution in the $l$ th cycle.

Table 1 Local operation times of SCCPs (Fig. 2)

\begin{tabular}{lccccccc}
\hline Streams & $i, k$ & $t_{i, 1}^{k}$ & $t_{i, 2}^{k}$ & $t_{i, 3}^{k}$ & $t_{i, 4}^{k}$ & $t_{i, 5}^{k}$ & $t_{i, 6}^{k}$ \\
\hline$P_{1}^{1}$ & 1,1 & 2 & 2 & 2 & 2 & - & - \\
$P_{2}^{1}$ & 2,1 & 2 & 2 & 2 & - & - & - \\
$P_{3}^{1}$ & 3,1 & 3 & 3 & 3 & 3 & 3 & - \\
$P_{4}^{1}$ & 4,1 & 1 & 1 & 1 & 1 & 1 & 1 \\
$P_{4}^{2}$ & 4,2 & 1 & 1 & 1 & 1 & 1 & 1 \\
$P_{5}^{1}$ & 5,1 & 2 & 2 & 2 & 2 & 2 & - \\
$P_{5}^{2}$ & 5,2 & 1 & 1 & 1 & 1 & 1 & - \\
$P_{6}^{1}$ & 6,1 & 1 & 1 & 1 & - & - & - \\
\hline
\end{tabular}

- $\Theta=\left\{\sigma_{1}, \sigma_{2}, \ldots, \sigma_{c}, \ldots, \sigma_{m}\right\}$ is the set of priority dispatching rules, where $\sigma_{c}=\left(s_{c, 1}, \ldots, s_{c, d}, \ldots, s_{c, l p(c)}\right)$ are sequence components which determine the order in which the processes can be executed on the resource $R_{c}$, where $s_{c, d} \in H$ ( $H$ is the set of streams, .e.g., for Fig. $\left.2, H=\left\{P_{1}^{1}, P_{2}{ }^{1}, P_{3}{ }^{1}, P_{4}{ }^{1}, P_{4}{ }^{2}, P_{5}{ }^{1}, P_{5}{ }^{2}, P_{6}{ }^{1}\right\}\right)$.

Using the above notation, an SCCP can be defined as a tuple:

$S C=((R, S L), S M)$,

where:

$R=\left\{R_{1}, R_{2}, \ldots, R_{c}, \ldots, R_{m}\right\}$ - the set of resources, where $m$ is the number of resources

$S L=\left(S T_{L}, B E_{L}\right)$ - the structure of local processes, i.e.

$S T_{L}=(U, T)$ - the variables describing the layout of local processes

$U=\left\{p_{1}^{1}, \ldots, p_{1}^{l s(1)}, \ldots, p_{n}^{1}, \ldots, p_{n}^{l s(n)}\right\}$ - the set of routes of local process where $l s(i)$ is the number of streams belonging to the process $P_{i}$ and $n$ is the number of local processes

$$
T=\left\{t_{1}^{1}, \ldots, t_{1}^{l s(1)}, \ldots, t_{n}^{1}, \ldots, t_{n}^{l s(n)}\right\} \text { - the set of }
$$
sequences of operation times in local processes.

$B E_{L}=(\Theta, \Psi, \Xi)$ - the variables describing the behavior of local processes

$\Theta=\left\{\sigma_{1}, \sigma_{2}, \ldots, \sigma_{c}, \ldots, \sigma_{m}\right\}$ - the set of priority dispatching rules

$\Psi=\left(\psi_{1}, \psi_{2}, \ldots, \psi_{i}, \ldots, \psi_{n}\right)$ - the sequence of relative frequencies of mutual executions of local processes

$\Xi$ - the number of occurrences of $\Psi$ in a cyclic steady state.

$S M=\left(S T_{M}, B E_{M}\right)$ - the structure of multimodal processes, i.e.

$S T_{M}=(M, T)$ - the variables describing the layout of the level of a multimodal process, where $M=\left\{m p_{1}, \ldots\right.$, $\left.m p_{i}, \ldots, m p_{w}\right\}$ is the set of routes of a multimodal process and $w$ is the number of multimodal processes $m P_{i}$

$m T=\left\{m t_{1} \ldots m t_{w}\right\}$ - the set of sequences of operation times in multimodal processes.

$B E_{M}=(m \Psi, m \Xi)$ - the variables describing the behavior of multimodal processes

$m \Psi=\left(m \psi_{1}, m \psi_{2}, \ldots, m \psi_{i}, \ldots, m \psi_{w}\right)$ - the sequence of relative frequencies of mutual executions of multimodal processes

$m \Xi$ the number of occurrences of $m \Psi$ in a cyclic steady state.

The SCCP model (2) can be seen as a multilevel model, cf. Fig. 3, i.e., a model composed of an " $R$ level" 
Fig. 3 Multilayered model of the behavior of an SCCP (see the SCCP in Fig. 2)

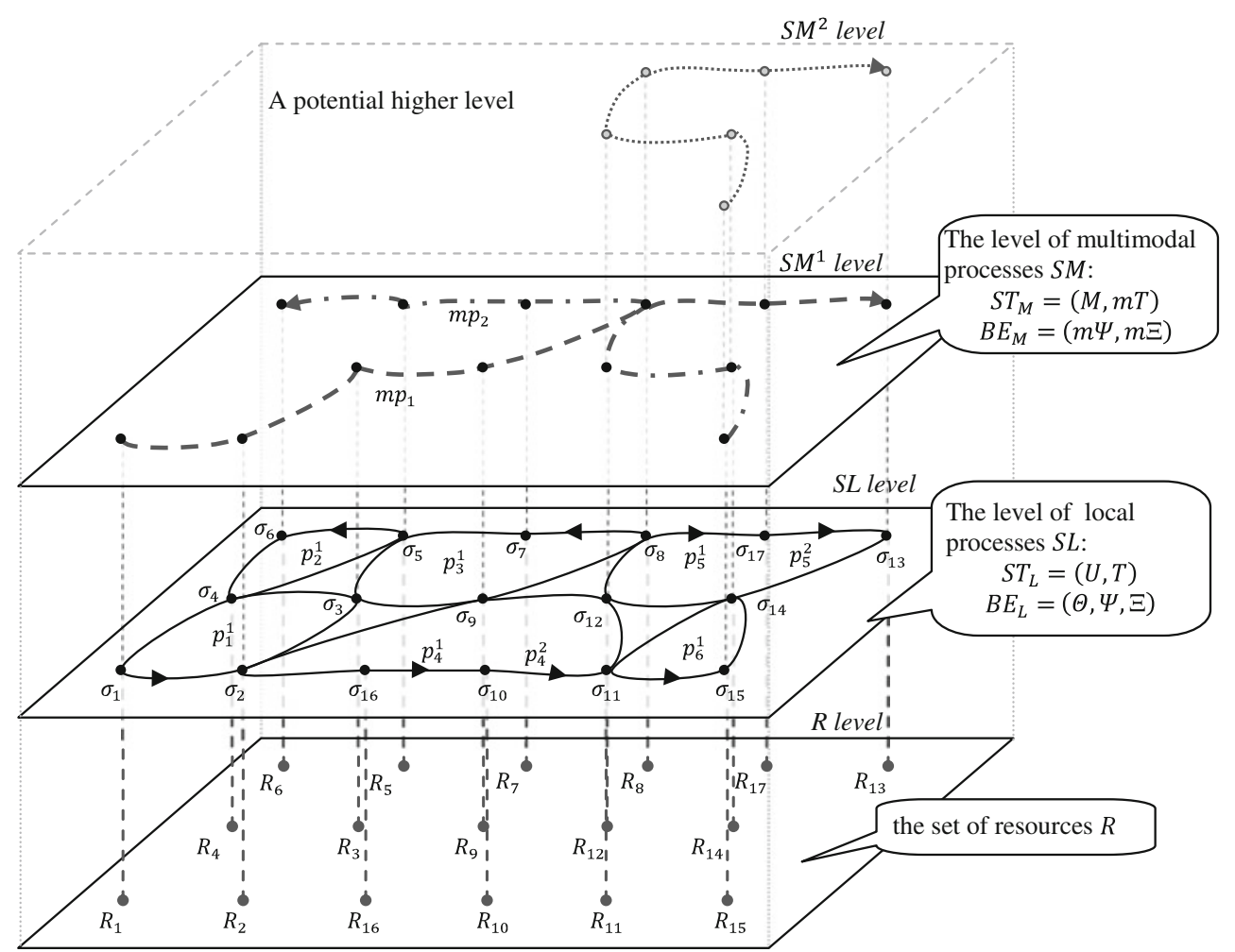

(resources), an "SL level" (local cyclic processes), and an "SM level" (multimodal cyclic processes), as well as an "SM level" (the $i$ th meta-multimodal process). The $S L$ level determines the structure of transportation routes of local processes $U$, as well as the parameters $\Theta, \Psi$, and $\Xi$ which specify the required behavior of the system. In turn, the $S M^{1}$ level takes into account multimodal, processes, as well as meta-multimodal processes $\left(S M^{2}\right.$ level $)$ composed of multimodal processes from the $S M^{1}$ level. In other words, we assume that the variables describing $S M^{i}$ are the same as in the case of $S M$, whereas the routes of the multimodal process of the $i$ th level remain composed of the processes from the $(i-1)$ th level. The presented model is an extended version of a simplified model limited to $R$ and $S L$ levels, which is introduced in $[3,5]$.

Therefore, in general, the $S C=((R, S L), S M)$ model can be seen as composed of $i$ levels:

$\left.S C^{i}=\left(\left(\left((R, S L), S M^{1}\right), S M^{2}\right), \ldots\right), S M^{i}\right)$.

Note that the cyclic behavior of $S C^{i-1}$ implies the periodic behavior of $S C^{i}$.

\subsection{Problem formulation}

Let us consider an SCCP specified by a given set $R$ of resources, dispatching rules $\Theta$, the routes of local and multimodal processes $U$ and $M$, respectively, and an initial allocation of processes. The most important issue here is the periodicity of the SCCP, i.e., does there exist a cyclic execution of local processes? And if so, what is the period $T c$ ? Further questions are concerned with cyclic execution of multimodal processes.

In order to find the answers to the above questions, more detailed questions must be answered first. Which allocations of initial processes are admissible (i.e., which AGV dockings are possible)? Which dispatching rules $\Theta$ assure the periodicity of a given SCCP (in the local and multimodal sense), while retaining the assumed frequency $(\Psi, m \Psi)$ of the execution of processes within the global period (local $T c$ and multimodal $T m$ )? In general, however, apart from the above formulations of forward problems, inverse problems can be considered as well. For instance, does there exist an SCCP structure of a local $(S L)$ and/or multimodal layer $(S M)$, such that the assumed steady cyclic state can be achieved?

In the rest of the paper, the following forward problem is considered:

Given an SCCP defined by $S C$ (2), i.e., $R, S L=\left(S T_{L}\right.$, $\left.B E_{L}\right), S M=\left(S T_{M}, B E_{M}\right)$,

is it possible to attain cyclic behavior of $S C$ (i.e., the encompassing cyclic steady states of local and multimodal processes)?

In other words, assuming the declarative approach, we are searching for the CSP providing a formal framework aimed at prototyping dispatching rules for the considered SCCP. 


\section{Refinement of the space of cyclic steady states}

\subsection{Generation of state space}

Let us consider the following definition of an SCCP state, which describes the allocation of both local and multimodal processes:

$S^{k}=\left(S l^{r}, M A^{k}\right)$,

where:

- $\quad S l^{r}$ - the $r$ th state of local processes, corresponding to the $k$ th state of multimodal processes,

$S l^{r}=\left(A^{r}, Z^{r}, Q^{r}\right)$,

where:

$A^{r}=\left(a_{1}{ }^{r}, a_{2}{ }^{r}, \ldots, a_{c}{ }^{r}, \ldots, a_{m}{ }^{r}\right)$ _allocation of processes in the $r$ th state, in which $a_{c}{ }^{r} \in P \cup\{\Delta\}, a_{c}{ }^{r}=P_{i}^{k}$, the $c$ th resource $R_{c}$, is occupied by the local stream $P_{i}^{k}$ and $a_{c}{ }^{r}=\Delta$, the $c$ th resource $R_{c}$, is unoccupied.

$Z^{r}=\left(z_{1}^{r}, z_{2}^{r}, \ldots, z_{c}^{r}, \ldots z_{m}^{r}\right)$ - the sequence of semaphores corresponding to the $r$ th state, in which $z_{c}{ }^{r}=P_{i}^{k}$ is the name of the stream (specified in the $c$ th dispatching rule $\sigma_{c}$, allocated to the $c$ th resource) which was allowed to occupy the $c$ th resource, e.g., $z_{c}{ }^{r}=P_{i}^{k}$ means that stream $P_{i}^{k}$ is currently allowed to occupy the $c$ th resource

$Q^{r}=\left(q_{1}{ }^{r}, q_{2}{ }^{r}, \ldots, q_{c}{ }^{r}, \ldots, q_{m}{ }^{r}\right)$ - the sequence of semaphore indices, corresponding to the $r$ th state, in which $q_{c}{ }^{r}$ determines the position of the semaphore $z_{c}{ }^{r}$ in the priority dispatching rule $\sigma_{c}, z_{c}{ }^{r}=s_{c,\left(q_{c}{ }^{r}\right)}, q_{c}{ }^{r} \in \mathbb{N}$. For instance, $q_{2}{ }^{r}=2$ and $z_{2}{ }^{r}=P_{1}^{2}$ correspond to the semaphore $z_{2}{ }^{r}=P_{1}^{2}$ taking the second position in the priority dispatching rule $\sigma_{2}$.

- $M A^{k}$ - the sequence of allocations of multimodal processes: $M A^{k}=\left(m A_{1}^{k}, \ldots, m A_{u}^{k}\right)$, in which $m A_{i}^{k}$ is the allocation of the process $m P_{i}$, i.e.,

$m A_{i}^{k}=\left(m a_{i, 1}{ }^{k}, m a_{i, 2}{ }^{k}, \ldots, m a_{i, c}{ }^{k}, \ldots, m a_{i, m}{ }^{k}\right)$,

where $m$ is the number of resources $R, m a_{i, c}{ }^{k} \in\left\{m P_{i}, \Delta\right\}$, $m a_{i, c}{ }^{k}=m P_{i}$ which means that the $c$ th resource $R_{c}$ is occupied by the $i$ th multimodal process $P_{i}$, and $m a_{i, c}{ }^{k}=\Delta$, the $c$ th resource $R_{c}$, is released by the $i$ th multimodal process $m P_{i}$.

In this context, the state $S^{k}$ is feasible [5] when:

- Semaphores of occupied resources indicate the streams allocated to those resources,

- Each local/multimodal stream is allotted to a unique resource due to a relevant local/multimodal process route.
The introduced concept of the $k$ th state $S^{k}$ enables to create a space $\mathbb{S}$ of feasible states. To illustrate this, let us consider the state space of an SCCP composed of six resources and three local cyclic processes supporting one multimodal process (see Fig. 4). The observed behavior is twofold, i.e., the levels of local $S L$ and multimodal $S M$ processes can be distinguished. In the case of the level of local processes, the states $S l^{i}$ are denoted by "filled circles," and in the case of the level of multimodal processes, the relevant states $S^{i} \in \mathbb{S}$ are denoted by "unfilled circles." States $S l^{j} \in \mathbb{S} l$ can be considered as a part of associated states $S^{i} \in \mathbb{S}$, i.e., the states that are elevated versions of relevant states $S^{i}$. The transitions linking feasible states $S^{k}, S^{l} \in \mathbb{S}$, while following the constraints of nonpreemption and mutual exclusion, are denoted by $S^{k} \rightarrow S^{l}$, and they encompass the next-state function $\delta: S^{l}=\delta\left(S^{k}\right)$, the definition of which [3] leads to the following property:

Each $S^{i} \in \mathbb{S}$ can be preceded by a subset of states $\mathbb{S P}^{i}$, $\mathbb{S P}^{i} \subset \mathbb{S}\left(\right.$ also $\left.\mathbb{S P}^{i}=\emptyset\right)$, i.e., $\forall S^{k} \in \mathbb{S P}^{i}, S^{i}=\delta\left(S^{k}\right)$ but can result only in a unique state $S^{j} \in \mathbb{S}$, i.e., there exists at most one $S^{j} \in \mathbb{S}, S^{j}=\delta\left(S^{i}\right)$.

The deadlock state $S^{*} \in \mathbb{S}$ resulting in an SCCP blockade is free from any descendent state. In this context, two types of steady state behaviors can be considered: a cyclic steady state and a deadlock state.

The set $m S c^{*}=\left\{S^{k_{1}}, S^{k_{2}}, S^{k_{3}}, \ldots, S^{k_{v}}\right\}, m S c^{*} \subset \mathbb{S}$ is called the reachability state space of multimodal processes generated by an initial state $S^{k_{1}} \in \mathbb{S}$, if the following condition holds:

$S^{k_{1}} \stackrel{i-1}{\longrightarrow} S^{k_{i}} \stackrel{v-i-1}{\longrightarrow} S^{k_{v}} \longrightarrow S^{k_{i}}$

where $S^{a} \stackrel{i}{\rightarrow} S^{b}$ - the transition defined in [5],

$S^{k_{1}} \stackrel{i}{\rightarrow} S^{k_{i+1}} \equiv S^{k_{1}} \rightarrow S^{k_{2}} \rightarrow S^{k_{3}} \rightarrow \ldots \rightarrow S^{k_{i+1}}$.

The set $m S c=\left\{S^{k_{i}}, \quad S^{k_{i+1}}, \ldots, \quad S^{k_{v}}\right\}, m S c \subseteq m S c^{*}$, is called the cyclic steady state of multimodal processes (i.e., a cyclic steady state of an SCCP) with a period $T m=\|m S c\|, T m>$ 1. In other words, the cyclic steady state contains such a set of states, where starting from any selected state, it is possible to reach the remaining states and finally reach this selected state again:

$\forall_{S^{k} \in m S c}\left(S^{k \stackrel{T m-1}{\rightarrow}} S^{k}\right)$

The cyclic steady state $S c$ specified by the period $T c$ of the execution of local processes is defined in a similar way. Graphically, the cyclic steady states $S c$ and $m S c$ are described by cyclic and spiral digraphs, respectively (see Fig. 4). Moreover, since an initial state $S^{k_{1}} \in \mathbb{S}$ either leads to $m S c$ or to a deadlock state $S^{*}$, i.e., $S^{k_{1}} \stackrel{i-1}{\longrightarrow} S^{k_{i}} \stackrel{v-i-1}{\longrightarrow} S^{k_{v}} \rightarrow S^{*}$, multimodal processes can also reach a deadlock state, denoted by "circles with a cross in the middle" in Fig. 4. 
Fig. 4 Space of feasible states encompassing behavior of the SCCP from Fig. 2

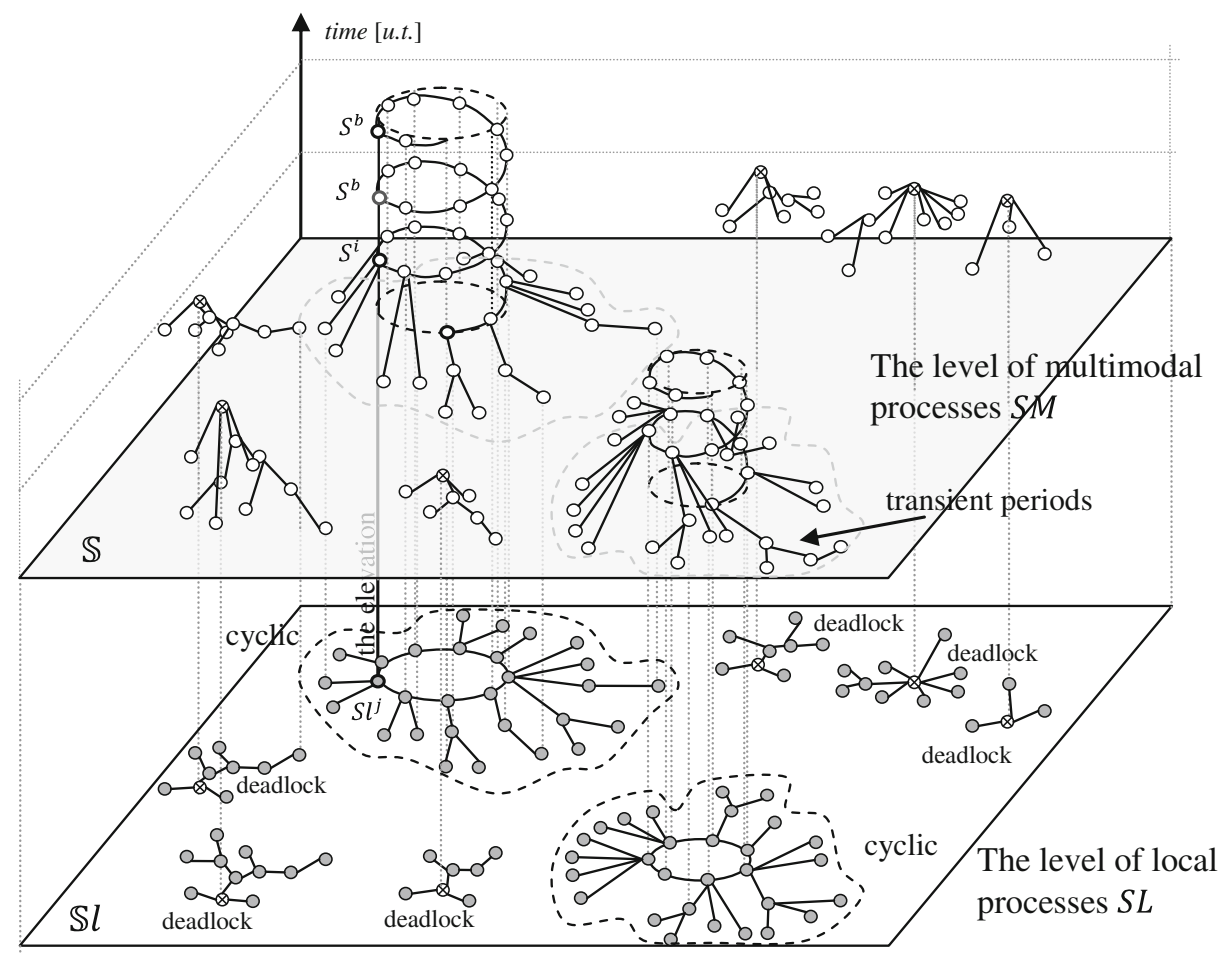

Legend:

$\begin{array}{cc}-i \text {-th local state } & 0-i \text {-th multimodal state } \\ S l^{i}=\left(A^{i}, Z^{i}, Q^{i}\right) & S^{i}=\left(S l^{j}, M A^{i}\right)\end{array}$

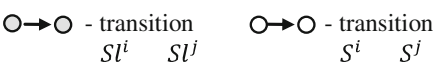

$\otimes$ - deadlock state

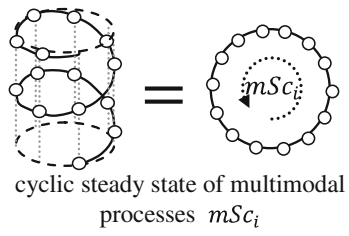

In this context, the problem formulated in Section 2.2 can be stated as follows: Given an SCCP defined by $S C$ (2), i.e., $R$, $S L=\left(S T_{L}, B E_{L}, S E_{L}\right), M=\left(S T_{M}, B E_{M}, S E_{M}\right)$, does there exist a cyclic steady state $m S c$ in the state space $S$ of the given SCCP?

Note that the above question gives rise to the following question: Does there exist an initial state $S^{0}$ that would generate the cyclic steady state $m S c$ ? This means that searching for a cyclic steady state $m S c$ in a given SCCP can be seen as a reachability problem where for an assumed initial state $S^{0}$ (i.e., for selected allocations of local and multimodal processes), we look for a state $S^{k}$ such that $S^{0} \stackrel{i}{\rightarrow} S^{k} \stackrel{T m-1}{\longrightarrow} S^{k}$ holds.

Refining the space of cyclic steady states from a given space of feasible states does not pose difficulty. However, the problem of generating the space of feasible states is NP-hard. The majority of states either end in deadlocks or lead to deadlock states. Therefore, in order to avoid the generation of the entire space of feasible states, let us focus on an alternative approach aimed at generating cyclic steady states.

\subsection{Generation of the space of cyclic steady states}

Since the parameters describing an SCCP are usually discrete, and the relations between them can be seen as constraints, the cyclic scheduling problems that involve them can be presented in the form of a CSP [2, 5, 17]. More formally, CSP is a framework for solving combinatorial problems specified by pairs: a set of variables and associated domains and a set of constraints restricting the possible combinations of variable values. Thus, in the case of $S C$ (2), the CSP is defined as follows:

$C S(S C)=\left(\left(\{X, T c, m X, T m\},\left\{D_{X}, D_{T c}, D_{m X}, D_{T m}\right\}\right), C\right)$,

where:

$X, T c, m X, T m$ - the decision variables, where $T c$ and $T m$ are the local and multimodal periodicities;

$X=\left\{X_{1}^{1}, \ldots, X_{1}^{l s(1)}, \ldots, X_{n}^{1}, \ldots, X_{n}^{l s(n)}\right\}$ is the set of sequences of $X_{i}^{k}$, and $X_{i}^{k}=\left(x_{i, 1,1}^{k}, \ldots, x_{i}^{k}, \operatorname{lr}(i), 1, \ldots\right.$, $\left.x_{i, 1, \Xi}^{k}, \ldots, x_{i, l r(i), \Xi}^{k}\right)$ where $x_{i, j, q}^{k}$ is the moment when the operation $o_{i, j}^{k}$ (local process) begins in the first cycle, whereas $x_{i, j, q}^{k}$ and $x_{i, j, q}^{k}(l)$ are linked by $x_{i, j, q}^{k}(l)=x_{i, j, q}^{k}+$ $l \cdot T c, l \in \mathbb{Z}, T c=x_{i, j, q}^{k}(l+1)-x_{i, j, q}^{k}(l)$.

Analogously, $m X=\left\{m X_{1}, m X_{2} \ldots, m X_{i}, \ldots, m X_{w}\right\}$ is the set of sequences of $m X_{i}$, and $m X_{i}=\left(m x_{i, 1,1}, \ldots, m\right.$ 
$x_{i, \operatorname{lm}(i), 1}, \ldots, m x_{i, 1, \Xi}, \ldots, m x_{i, \operatorname{lm}(i) \Xi,)}$ where $m x_{i, 1,1}$ is the moment when the operation $m o_{i, j}$ (of a multimodal process) begins in the first cycle $m x_{i, j, k}(l)=m x_{i, j, k}+$ $l \cdot T m$, and $T m=m x_{i, j, k}(l+1)-m x_{i, j, k}(l)$.

The following domains of decision variables are considered:

$D_{X}, D_{m X}$ the family of sets of admissible entry values

$X_{i}, x_{i, j, q}^{k} \in \mathbb{Z}$ and $m X_{i}, m x_{i, j, k} \in \mathbb{Z}$

$D_{T c}, D_{T m}$ - the domains of the variable $T c \in \mathbb{N}$ and $T m$ $\in \mathbb{N}$.

$C$ constraints are specified by both:

- $e p_{i, j, q}^{k}\left(S T_{L}, B E_{L}\right)$ the set of constraints (equations) linking $S T_{L}$ (structure of local processes) and $B E_{L}$ (behavior of local processes). Each $e p_{i, j, q}^{k}\left(S T_{L}, B E_{L}\right)$ describes the temporal relation (in accordance with the conditions presented above [5]) between the moments when the operation begins during its $q$ th execution: $i=1, . ., n$; $j=1, \ldots, \operatorname{lr}(i) ; k=1, . ., l s(i) ;$ and $\mathrm{q}=1, \ldots, \Xi$.

- $\quad e q_{i, j, k}\left(S T_{M}, B E_{M}\right)$ the set of constraints (equations) linking $S T_{M}$ (structure of multimodal processes) and $B E_{M}$ (behavior of multimodal processes). Each $e q_{i, j, k}\left(S T_{M}, B E_{M}\right)$ describes the temporal relation between the moments when the multimodal operations begin during their $k$ th execution: $i=1, . ., w ; j=1, \ldots, \operatorname{lm}(i) ;$ and $\mathrm{k}=1, \ldots, \mathrm{m} \Xi$.

In other words, the problem discussed in Section 2.2 boils down to the following: Given an SCCP described by $C S(S C)(9)$ (i.e., the constraint satisfaction problem determined by $S C$ ), do there exist $X, T c, m X$, and $T m$ whose values satisfy all the constraints $C$ ?

The solution to the problem (9) provides us with sets of sequences $X$ and $m X$, whose values guarantee the required cyclic behavior of the SCCP while keeping the set of constraints $C$ satisfied. The constraints $C$ that can be seen as conditions sufficient for the cyclic behavior of the SCCP (resulting in a collision-free and deadlock-free [12] execution of processes) are formulated using the operator max, which takes into account the pipeline nature of the flow of local processes. The application of the max operator can be seen as an extension of the max constraint concept introduced in [5].

Constraints on local processes In order to explain how the constraints of local processes are designed, let us consider an example of an SCCP shown in Fig. 5. The operation $o_{1,3}^{1}$ (executed by $P_{1}^{1}$ on the resource $R_{5}$ ) can be started (i.e., begin its first execution; $q=1$ ) only if the preceding operation $o_{1,2}^{1}$ (executed by $P_{1}^{1}$ on $R_{3}$ ) has been completed $\left(x_{1,2,1}^{1}+t_{1,2}^{1}\right)$ and the resource $R_{5}$ has been released, i.e., if the stream $P_{3}^{1}$ occupying the resource $R_{5}$ starts its subsequent operation at $x_{3,3,1}^{1}+1$. Thus, the considered relation $e p_{1,3,1}^{1}$ $\left(S T_{L}, B E_{L}\right)$ can be specified by the following formulae:

$x_{1,3,1}^{1}=\max \left\{\left(x_{3,3,1}^{1}+1\right) ;\left(x_{1,2,1}^{1}+t_{1,2}^{1}\right)\right\}$,

where $x_{i, j, q}^{k}$ is the moment when the operation $o_{i, j}^{k}$ forms, the $P_{i}^{k}$ stream begins in its $q$ th execution.

Table 2 contains the remaining constraints describing the local processes of the SCCP from Fig. 5.

For all constraints, the following principle holds: the moment when the operation $o_{i, j}^{k}$ begins is calculated as a maximum of the completion time of operation $o_{i, j-1}^{k}$ preceding $o_{i, j}^{k}$, and the release time of the resource $p_{i, j}^{k}$ awaiting for $o_{i, j}^{k}$ execution.
Fig. 5 SCCP with dispatching rules: $\sigma_{1}=\left(P_{2}{ }^{1}, P_{3}{ }^{1}\right), \sigma_{3}=$ $\left(P_{2}^{1}, P_{1}^{1}\right), \sigma_{5}=\left(P_{3}{ }^{1}, P_{2}{ }^{1}\right)$, and $\Psi=(1,1,1, \Xi=1$

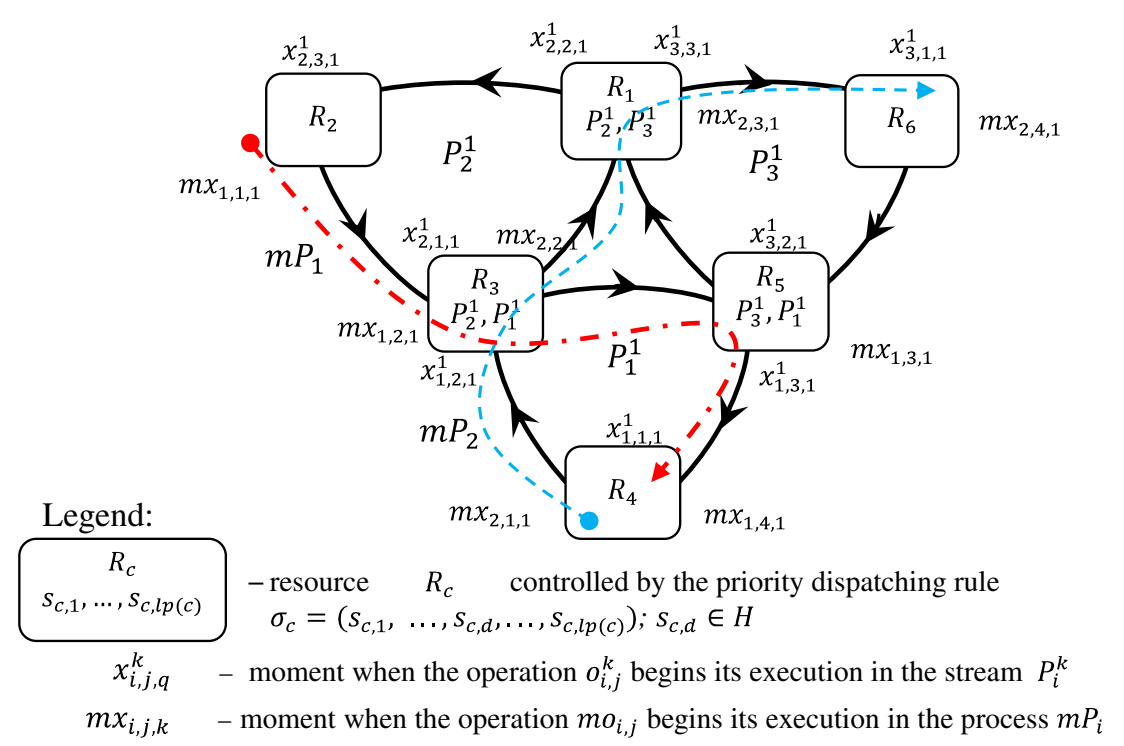


Table 2 Constraints of local processes determining the moments $x_{i, j, q}^{k}$ for the SCCP from Fig. 5

$$
\begin{array}{ll}
\hline x_{1,1,1}^{1}=\max \left\{\left(x_{1,3,1}^{1}+t_{1,3}^{1}-T c\right) ;\left(x_{1,3,1}^{1}+1-T c\right)\right\} & x_{1,2,1}^{1}=\max \left\{\left(x_{1,1,1}^{1}+t_{1,2}^{1}\right) ;\left(x_{2,2,1}^{1}+1\right)\right\} \\
x_{2,2,1}^{1}=\max \left\{\left(x_{2,1,1}^{1}+t_{2,1}^{1}\right) ;\left(x_{3,1,1}^{1}+1\right)\right\} & x_{2,1,1}^{1}=\max \left\{\left(x_{2,3,1}^{1}+t_{2,3}^{1}-T c\right) ;\left(x_{1,3,1}^{1}+1-T c\right)\right\} \\
x_{3,1,1}^{1}=\max \left\{\left(x_{3,3,1}^{1}+t_{3,3}^{1}-T c\right) ;\left(x_{3,3,1}^{1}+1-T c\right)\right\} & \left.x_{2,3,1}^{1}=\max \left\{\left(x_{2,2,1}^{1}+t_{2,2}^{1}\right) ;\left(x_{2,2,1}^{1}+1\right\}\right)\right\} \\
x_{3,3,1}^{1}=\max \left\{\left(x_{2,3,1}^{1}+1-T c\right) ;\left(x_{3,2,1}^{1}+t_{3,2}^{1}\right)\right\} & x_{3,2,1}^{1}=\max \left\{\left(x_{3,1,1}^{1}+t_{3,1}^{1}\right) ;\left(x_{1,1,1}^{1}+1\right)\right\} \\
\hline
\end{array}
$$

$e p_{i, j, q}^{k}\left(S T_{L}, B E_{L}\right):$

moment when the operation $\mathrm{o}_{\mathrm{i}, \mathrm{j}}^{\mathrm{k}}$ begins in its $\mathrm{q}-$ th execution $=$ $=\max \left\{\right.$ moment when $p_{i, j}^{k}$ is released,

, moment when the operation $\mathrm{o}_{\mathrm{i}, \mathrm{j}-1}^{\mathrm{k}}$ completes $\}$,

$$
i=1, . ., n ; j=1, \ldots, \operatorname{lr}(i) ; k=1, . ., l s(i) ; q=1, \ldots, \Xi
$$

Therefore, following the constraints (11) guarantees a deadlock-free execution of cyclic processes at the $S L$ level (see Fig. 3).

Note that the constraints (11) indirectly take into account the assumed dispatching priority rules $\Theta$ and routes of streams $U$. This means that the SCCP being free of deadlock depends on the dispatching rules $\Theta$ determining the operations executed on the shared resource, i.e., the satisfiability of constraints (11). This is because the moment when the operation $o_{i, j-1}^{k}$ completes is determined by the route of the stream $p_{i}^{k}$ and, in turn, the moment when $p_{i, j}^{k}$ is released depends on the operation executed before $o_{i, j}^{k}$ by $p_{i, j}^{k}$.

Constraints on multimodal processes The constraints determining the execution of local cyclic processes have already been discussed in depth in [3-5]. Multimodal processes, however, have not yet been discussed in a similar way. Therefore, for the sake of simplicity, let us assume that multimodal processes are collision- and deadlock-free. At the same time, multimodal processes can occupy the same resource and may use the same local process for their execution.

The research presented in $[3,4]$ focuses on constraints determining cyclic steady state behavior and particularly on the conditions behind the concept of the next-state function. The lack of guarantee that any cyclic steady state is reachable from a given initial state was the main disadvantage of the results obtained so far. Moreover, because the number of potential initial states grows exponentially with the number of local processes, any real-life implementation of the results is rather limited. In turn, the conditions obtained in [5], which guarantee that an initial state belongs to a cyclic steady state, were limited to local cyclic processes only.

The constraints describing the relationship between the moments when successive operations begin are shown using the example of $m P_{1}$ from Fig. 5. The considered process executes on the set of the following resources: $R_{2}, R_{3}, R_{5}$, and $R_{4}$. The local cyclic process $P_{2}^{1}$ supports the execution of $m P_{1}$ between $R_{2}$ and $R_{3}$, whereas $P_{1}^{1}$ supports the execution of $m P_{1}$ between $R_{3}, R_{5}$, and $R_{4}$. This means that the process $m P_{1}$ can execute its operations only when the relevant operations from local processes are performed (i.e., $P_{1}^{1}$ or $P_{2}^{1}$ ). For instance, the operation $m o_{1,2}$ executed on $R_{3}$ depends on $P_{2}^{1}$. The starting moment $m x_{1,2,1}$ of the operation $m o_{1,2}$ coincides with the starting moment $\left(x_{2,2,1}^{1}+a \cdot T c\right)$, i.e., with the beginning of the operation $o_{2,2}^{1}$ and is executed after the completion of the preceding operation $m o_{1,2}$ (i.e., the operation $m o_{1,1}$ completed at the moment $\left.m x_{1,1,1}+m t_{1,1,1}\right)$. The constraint specifying this relationship has the following form:

$m x_{1,2,1}=\min \left\{\left(x_{2,2,1}^{1}+a \cdot T c\right) \mid a \in \mathbb{Z}, x_{2,2,1}^{1}+a \cdot T c \geq m x_{1,1,1}+m t_{1,1,1}\right\}$,

where:

$x_{i, j, q}^{k}$ - the moment when the operation $o_{i, j}{ }^{k}$ begins in the $q$ th execution of a local process

$m x_{i, j, q}$ the moment when the operation $m o_{i, j}$ begins in the $q$ th execution of a multimodal process

$T c$ - the periodicity of local cyclic steady states.

Table 3 contains the remaining constraints describing the multimodal processes of the SCCP from Fig. 5.

For all the constraints, the following principle holds: the moment when the operation $m o_{i, j}$ begins is the earliest moment when the operation of the local process can start (of course the one the multimodal process $m P_{i}$ requires), temporally following the moment when the operation $m o_{i, j-1}$ begins. More formally:

$e q_{i, j, k}\left(S T_{M}, B E_{M}\right):$

moment when the operation mo $_{i, j}$ begins $=$

$=\min \left\{\right.$ set of moments when the operation $o_{i j-1}^{k}$ begins, temporally following the moment when the previous operation mo $_{i, j-1}$ complees $\}$, $i=1, . ., w ; j=1, \ldots, \operatorname{lm}(i) ; k=1, \ldots, m \Xi$.

\section{Scheduling of periodic processes}

Let us consider the AGVS from Fig. 1 modeled in terms of SCCPs (see Fig. 2). Taking into account (2), it can be described as follows: 
Table 3 Constraints determining the moments $m x_{i, j, k}$ for the SCCP from Fig. 5

$$
\begin{aligned}
& \text { Multimodal process } m P_{1} \\
& m x_{1,1,1}=\min \left\{\left(x_{2,3,1}^{1}+a \cdot T c\right) \mid a \in \mathbb{Z}, x_{2,3,1}^{1}+a \cdot T c \geq m x_{1,4,1}+m t_{1,4,1}-T m\right\}, \\
& m x_{1,2,1}=\min \left\{\left(x_{2,2,1}^{1}+a \cdot T c\right) \mid a \in \mathbb{Z}, x_{2,2,1}^{1}+a \cdot T c \geq m x_{1,1,1}+m t_{1,1,1}\right\}, \\
& m x_{1,3,1}=\min \left\{\left(x_{1,3,1}^{1}+a \cdot T c\right) \mid a \in \mathbb{Z}, x_{1,3,1}^{1}+a \cdot T c \geq m x_{1,2,1}+m t_{1,2,1}\right\}, \\
& m x_{1,4,1}=\min \left\{\left(x_{1,1,1}^{1}+a \cdot T c\right) \mid a \in \mathbb{Z}, x_{1,1,1}^{1}+a \cdot T c \geq m x_{1,3,1}+m t_{1,3,1}\right\}
\end{aligned}
$$

Multimodal process $m P_{2}$

$$
\begin{aligned}
& m x_{2,1,1}=\min \left\{\left(x_{1,1,1}^{1}+a \cdot T c\right) \mid a \in \mathbb{Z}, x_{1,1,1}^{1}+a \cdot T c \geq m x_{2,4,1}+m t_{2,4,1}-T m\right\}, \\
& m x_{2,2,1}=\min \left\{\left(x_{1,2,1}^{1}+a \cdot T c\right) \mid a \in \mathbb{Z}, x_{1,2,1}^{1}+a \cdot T c \geq m x_{2,1,1}+m t_{1,2,1}\right\}, \\
& m x_{2,3,1}=\min \left\{\left(x_{2,2,1}^{1}+a \cdot T c\right) \mid a \in \mathbb{Z}, x_{2,2,1}^{1}+a \cdot T c \geq m x_{2,2,1}+m t_{2,2,1}\right\}, \\
& m x_{2,4,1}=\min \left\{\left(x_{3,1,1}^{1}+a \cdot T c\right) \mid a \in \mathbb{Z}, x_{3,1,1}^{1}+a \cdot T c \geq m x_{2,3,1}+m t_{2,3,1}\right\}, \\
& \bmod \{T m, T c\}=0
\end{aligned}
$$

Given $S C=((R, S L), S M)$, where:

$R=\left\{R_{1}, R_{2}, \ldots, R_{18}\right\}$ - the set of resources,

$S L=\left(S T_{L}, B E_{L}\right)$-the structure of local processes

$$
\begin{aligned}
& S T_{L}=(U, T) \\
& \qquad=\left\{p_{1}^{1}, p_{2}^{1}, p_{3}^{1}, p_{4}^{1}, p_{4}^{2}, p_{5}^{1}, p_{5}^{2}, p_{1}^{6}\right\} \\
& p_{1}^{1}=\left(R_{1}, R_{2}, R_{3}, R_{4}\right), p_{2}^{1}=\left(R_{4}, R_{5}, R_{6}\right), \\
& p_{3}^{1}=\left(R_{3}, R_{9}, R_{8}, R_{7}, R_{5}\right), \\
& p_{4}^{1}=p_{4}^{2}=\left(R_{2}, R_{16}, R_{10}, R_{11}, R_{12}, R_{9}\right), \\
& p_{6}^{1}=\left(R_{14}, R_{11}, R_{15}\right), \\
& p_{5}^{1}=p_{5}^{2}=\left(R_{8}, R_{17}, R_{13}, R_{14}, R_{12}\right)
\end{aligned}
$$

$T=\left\{t_{1}^{1}, t_{2}^{1}, t_{3}^{1}, t_{4}^{1}, t_{4}^{2}, t_{5}^{1}, t_{5}^{2}, t_{6}^{1}\right\}$, where the values of the elements of the sequences $t_{i}^{k}$ are given in Table 1

$B E_{L}=(\Theta, \Psi, \Xi)$

$$
\begin{aligned}
\Theta=\left\{\sigma_{1}, \sigma_{2}, \ldots, \sigma_{17}\right\} & \\
\sigma_{1} & =\left(P_{1}^{1}\right), \sigma_{2}=\left(P_{1}^{1}, P_{4}^{1}, P_{4}^{2}\right), \sigma_{3}=\left(P_{1}^{1}, P_{3}^{1}\right), \\
\sigma_{4} & =\left(P_{1}^{1}, P_{2}^{1}, P_{2}^{1}, P_{2}^{1}\right), \\
\sigma_{5} & =\left(P_{2}^{1}, P_{2}^{1}, P_{2}^{1}, P_{3}^{1}\right), \sigma_{6}=\left(P_{2}^{1}, P_{2}^{1}, P_{2}^{1}\right), \\
\sigma_{7} & =\left(P_{3}^{1}\right), \sigma_{8}=\left(P_{5}^{1}, P_{5}^{2}, P_{3}^{1}\right), \\
\sigma_{9} & =\left(P_{3}^{1}, P_{4}^{1}, P_{4}^{2}\right), \sigma_{10}=\left(P_{4}^{1}, P_{4}^{2}\right), \\
\sigma_{11} & =\left(P_{4}^{1}, P_{4}^{2}, P_{6}^{1}, P_{6}^{2}\right), \\
\sigma_{12} & =\left(P_{4}^{1}, P_{4}^{2}, P_{5}^{1}, P_{5}^{2}\right), \sigma_{13}=\left(P_{5}^{1}, P_{5}^{2}\right), \\
\sigma_{14} & =\left(P_{5}^{1}, P_{5}^{2}, P_{6}^{1}, P_{6}^{2}\right), \sigma_{15}=\left(P_{6}^{1}, P_{6}^{2}\right), \\
\sigma_{16} & =\left(P_{4}^{1}, P_{4}^{2}\right), \sigma_{17}=\left(P_{5}^{1}, P_{5}^{2}\right), \\
\Psi=(1,3,1,1,1,2) &
\end{aligned}
$$

$S M=\left(S T_{M}, B E_{M}\right)$-the structure of multimodal processes

$$
\begin{aligned}
& S T_{M}=(M, m T) \\
& M=\left\{m p_{1}, m p_{2}\right\} \\
& m p_{1}=\left(R_{1}, R_{2}, R_{3}, R_{9}, R_{8}, R_{17}, R_{13}\right), \\
& m p_{2}=\left(R_{15}, R_{14}, R_{12}, R_{8}, R_{7}, R_{5}, R_{6}\right) . \\
& m T=\left\{m t_{1}, m t_{2}\right\} \text {, where the values of all } \\
& \text { elements } m_{i} \text { are equal to } 1 \text { unit of time } \\
& B E_{M}=(m \Psi, m \Xi) \\
& m \Psi=(1,1) \\
& m \Xi=1
\end{aligned}
$$

The response to the following question is sought: Does there exist a cyclic behavior of the $S C$ (i.e., resulting in cyclic steady states of local and multimodal processes)?

The approach aimed at generating the space of feasible states (see Fig. 4) is time consuming (it is an NP-hard problem) and results mainly in a deadlock or in states leading to a deadlock. An alternative approach, based on the CSP formulation of the CS (9), enables to focus on (and, if possible, to generate) the dedicated cyclic steady states of both local and multimodal processes.

\subsection{Scheduling of repetitive processes}

Consider a given SCCP and its SL level. Searching for a possible cyclic steady state of local processes formulated in terms of a CSP can be stated as the following constraint satisfaction problem:

$C S(S C)=\left(\left(\{X, T c\},\left\{D_{X}, D_{T c}\right\}\right), C\right)$, 
Table 4 Constraints determining the moments $x_{i, j . q}^{k}$ when operations begin for the SCCP from Fig. 2

$$
\begin{aligned}
& \text { Local process } P_{1}^{1} \\
& x_{1,1,1}^{1}=\max \left\{\left(x_{1,4,1}^{1}+t_{1,4}^{1}-T c\right) ;\left(x_{1,4,1}^{1}+1-T c\right)\right\} \\
& x_{1,2,1}^{1}=\max \left\{\left(x_{1,1,1}^{1}+t_{1,1}^{1}\right) ;\left(x_{4,2,1}^{2}+1\right)\right\} \\
& x_{1,3,1}^{1}=\max \left\{\left(x_{1,2,1}^{1}+t_{1,2}^{1}\right) ;\left(x_{3,1,1}^{1}+1\right)\right\} \\
& x_{1,4,1}^{1}=\max \left\{\left(x_{1,3,1}^{1}+t_{1,3}^{1}\right) ;\left(x_{2,2,3}^{1}+1\right)\right\}
\end{aligned}
$$

Local process $P_{3}^{1}$

$$
\begin{aligned}
& x_{3,1,1}^{1}=\max \left\{\left(x_{3,5,1}^{1}+t_{3,5}^{1}-T c\right) ;\left(x_{1,4,1}^{1}+1\right)\right\} \\
& x_{3,2,1}^{1}=\max \left\{\left(x_{3,1,1}^{1}+t_{3,1}^{1}\right) ;\left(x_{4,1,1}^{2}+1\right)\right\} \\
& x_{3,3,1}^{1}=\max \left\{\left(x_{3,2,1}^{1}+t_{3,2}^{1}\right) ;\left(x_{5,2,1}^{2}+1\right)\right\} \\
& x_{3,4,1}^{1}=\max \left\{\left(x_{3,3,1}^{1}+t_{3,3}^{1}\right) ;\left(x_{3,5,1}^{1}+1-T c\right)\right\} \\
& x_{3,5,1}^{1}=\max \left\{\left(x_{3,4,1}^{1}+t_{3,4}^{1}\right) ;\left(x_{2,3,3}^{1}+1\right)\right\}
\end{aligned}
$$

Local process $P_{4}^{2}$

$$
\begin{aligned}
& x_{4,1,1}^{2}=\max \left\{\left(x_{4,6,1}^{2}+t_{4,6}^{2}-T c\right) ;\left(x_{4,2,1}^{1}+1\right)\right\} \\
& x_{4,2,1}^{2}=\max \left\{\left(x_{4,1,1}^{2}+t_{4,1}^{2}\right) ;\left(x_{4,3,1}^{1}+1\right)\right\} \\
& x_{4,3,1}^{2}=\max \left\{\left(x_{4,2,1}^{2}+t_{4,2}^{2}\right) ;\left(x_{4,4,1}^{1}+1\right)\right\} \\
& x_{4,4,1}^{2}=\max \left\{\left(x_{4,3,1}^{2}+t_{4,3}^{2}\right) ;\left(x_{4,2,3}^{1}+1\right)\right\} \\
& x_{4,5,1}^{2}=\max \left\{\left(x_{4,5,1}^{2}+t_{4,5}^{2}\right) ;\left(x_{4,6,1}^{1}+1\right)\right\} \\
& x_{4,6,1}^{2}=\max \left\{\left(x_{4,5,1}^{2}+t_{4,5}^{2}\right) ;\left(x_{4,1,1}^{1}+1+T c\right)\right\}
\end{aligned}
$$

Local process $P_{5}^{2}$

$$
\begin{aligned}
& x_{5,1,1}^{2}=\max \left\{\left(x_{5,5,1}^{2}+t_{5,5}^{2}-T c\right) ;\left(x_{5,2,1}^{1}+1\right)\right\} \\
& x_{5,2,1}^{2}=\max \left\{\left(x_{5,1,1}^{2}+t_{5,1}^{2}\right) ;\left(x_{5,3,1}^{1}+1\right)\right\} \\
& x_{5,3,1}^{2}=\max \left\{\left(x_{5,1,1}^{2}+t_{5,1}^{2}\right) ;\left(x_{5,4,1}^{1}+1\right)\right\} \\
& x_{5,4,1}^{2}=\max \left\{\left(x_{5,3,1}^{2}+t_{5,3}^{2}\right) ;\left(x_{5,5,1}^{1}+1\right)\right\} \\
& x_{5,5,1}^{2}=\max \left\{\left(x_{5,4,1}^{2}+t_{5,4}^{2}\right) ;\left(x_{5,1,1}^{1}+1+T c\right)\right\}
\end{aligned}
$$

$$
\begin{aligned}
& \text { Local process } P_{2}^{1} \\
& x_{2,1,1}^{1}=\max \left\{\left(x_{2,3,3}^{1}+t_{2,3}^{1}-T c\right) ;\left(x_{1,1,1}^{1}+1+T c\right)\right\} \\
& x_{2,2,1}^{1}=\max \left\{\left(x_{2,1,1}^{1}+t_{2,1,1}^{1}\right) ;\left(x_{3,1,1}^{1}+1\right)\right\} \\
& x_{2,3,1}^{1}=\max \left\{\left(x_{2,2,1}^{1}+t_{2,2,1}^{1}\right) ;\left(x_{2,1,1}^{1}+1\right)\right\} \\
& x_{2,1,2}^{1}=\max \left\{\left(x_{2,3,1}^{1}+t_{2,3,1}^{1}\right) ;\left(x_{2,2,1}^{1}+1\right)\right\} \\
& x_{2,2,2}^{1}=\max \left\{\left(x_{2,1,2}^{1}+t_{2,1,2}^{1}\right) ;\left(x_{2,3,1}^{1}+1\right)\right\} \\
& x_{2,3,2}^{1}=\max \left\{\left(x_{2,2,2}^{1}+t_{2,2,2}^{1}\right) ;\left(x_{2,1,2}^{1}+1\right)\right\} \\
& x_{2,1,3}^{1}=\max \left\{\left(x_{2,3,2}^{1}+t_{2,3,2}^{1}\right) ;\left(x_{2,2,2}^{1}+1\right)\right\} \\
& x_{2,2,3}^{1}=\max \left\{\left(x_{2,1,3}^{1}+t_{2,1,3}^{1}\right) ;\left(x_{2,3,2}^{1}+1\right)\right\} \\
& x_{2,3,3}^{1}=\max \left\{\left(x_{2,2,3}^{1}+t_{2,2,3}^{1}\right) ;\left(x_{2,1,3}^{1}+1\right)\right\}
\end{aligned}
$$

Local process $P_{4}^{1}$

$$
\begin{aligned}
& x_{4,1,1}^{1}=\max \left\{\left(x_{4,6,1}^{1}+t_{4,6}^{1}-T c\right) ;\left(x_{1,3,1}^{1}+1\right)\right\} \\
& x_{4,2,1}^{1}=\max \left\{\left(x_{4,1,1}^{1}+t_{4,1}^{1}\right) ;\left(x_{4,3,1}^{2}+1-T c\right)\right\} \\
& x_{4,3,1}^{1}=\max \left\{\left(x_{4,2,1}^{1}+t_{4,2}^{1}\right) ;\left(x_{4,3,1}^{2}+1-T c\right)\right\} \\
& x_{4,4,1}^{1}=\max \left\{\left(x_{4,3,1}^{1}+t_{4,3}^{1}\right) ;\left(x_{6,2,2}^{1}+1-T c\right)\right\} \\
& x_{4,5,1}^{1}=\max \left\{\left(x_{4,4,1}^{1}+t_{4,4}^{1}\right) ;\left(x_{5,2,1}^{2}+1\right)\right\} \\
& x_{4,6,1}^{1}=\max \left\{\left(x_{4,5,1}^{1}+t_{4,5}^{1}\right) ;\left(x_{3,3,1}^{1}+1\right)\right\}
\end{aligned}
$$

Local process $P_{5}^{1}$

$$
\begin{aligned}
& x_{5,1,1}^{1}=\max \left\{\left(x_{5,5,1}^{1}+t_{5,5}^{1}-T c\right) ;\left(x_{3,4,1}^{1}+1\right)\right\} \\
& x_{5,2,1}^{1}=\max \left\{\left(x_{5,1,1}^{1}+t_{5,1}^{1}\right) ;\left(x_{5,3,1}^{2}+1-T c\right)\right\} \\
& x_{5,3,1}^{1}=\max \left\{\left(x_{5,2,1}^{1}+t_{5,2}^{1}\right) ;\left(x_{5,4,1}^{2}+1-T c\right)\right\} \\
& x_{5,4,1}^{1}=\max \left\{\left(x_{5,3,1}^{1}+t_{5,3}^{1}\right) ;\left(x_{6,1,1}^{1}+1\right)\right\} \\
& x_{5,5,1}^{1}=\max \left\{\left(x_{5,4,1}^{1}+t_{5,4}^{1}\right) ;\left(x_{4,6,1}^{2}+1\right)\right\}
\end{aligned}
$$

Local process $P_{6}^{1}$

$$
\begin{aligned}
& x_{6,1,1}^{1}=\max \left\{\left(x_{6,3,2}^{1}+t_{6,3}^{1}-T c\right) ;\left(x_{4,5,1}^{2}+1\right)\right\} \\
& x_{6,2,1}^{1}=\max \left\{\left(x_{6,1,1}^{1}+t_{6,1}^{1}\right) ;\left(x_{6,3,2}^{1}+1-T c\right)\right\} \\
& x_{6,3,1}^{1}=\max \left\{\left(x_{6,2,1}^{1}+t_{6,2}^{1}\right) ;\left(x_{5,5,1}^{2}+1\right)\right\} \\
& x_{6,1,2}^{1}=\max \left\{\left(x_{6,3,1}^{1}+t_{6,3}^{1}\right) ;\left(x_{6,2,1}^{1}+1\right)\right\} \\
& x_{6,2,2}^{1}=\max \left\{\left(x_{6,1,2}^{1}+t_{6,1}^{1}\right) ;\left(x_{6,3,1}^{1}+1\right)\right\} \\
& x_{6,3,3}^{1}=\max \left\{\left(x_{6,2,2}^{1}+t_{6,2}^{1}\right) ;\left(x_{6,4,1}^{1}+1\right)\right\}
\end{aligned}
$$


Table 5 Values of the moments $x_{i, j, q}^{k}$ when operations begin for the SCCP from Fig. 2

\begin{tabular}{lllllllllll}
\hline Starting & $x_{1,1,1}^{1}$, & $x_{2,1,1}^{1}$, & $x_{1,2,1}^{1}$, & $x_{3,1,1}^{1}$, & $x_{2,2,1}^{1}$, & $x_{4,4,1}^{2}$ & $x_{2,3,1}^{1}$, & $x_{1,3,1}^{1}$ & $x_{2,1,2}^{1}$ & $x_{3,3,1}^{1}$ \\
moments: & $x_{3,5,1}^{1}$, & $x_{5,1,1}^{2}$, & $x_{5,3,1}^{1}$, & $x_{4,4,1}^{1}$, & $x_{4,5,1}^{1}$, & & $x_{3,2,1}^{1}$ & & & \\
& $x_{5,2,1}^{1}$, & $x_{6,2,1}^{1}$ & $x_{6,3,1}^{1}$ & $x_{5,2,1}^{2}$, & $x_{4,3,1}^{2}$ & & & & \\
& $x_{6,1,1}^{1}$ & & & $x_{6,1,2}^{1}$ & & & & & & \\
Values: & 0 & 1 & 2 & 3 & 4 & 5 & 6 & 7 & 8 & 9 \\
Corresponding & $S l^{0}$ & $S l^{1}$ & $S l^{2}$ & $S l^{3}$ & $S l^{4}$ & $S l^{5}$ & $S l^{6}$ & $S l^{7}$ & $S l^{8}$ & $S l^{9}$ \\
states: & $x_{2,2,2}^{1}$, & $x_{4,1,1}^{1}$, & $x_{2,3,2}^{1}$, & $x_{4,3,1}^{1}$, & $x_{2,1,3}^{1}$, & $x_{5,5,1}^{1}$ & $x_{2,2,3}^{1}$, & $x_{1,4,1}^{1}$, & $x_{2,3,3}^{1}$, \\
Starting & $x_{4,5,1}^{1}$, & $x_{4,5,1}^{2}$ & $x_{3,4,1}^{1}$, & $x_{4,1,1}^{2}$, & $x_{4,2,1}^{2}$, & & $x_{5,4,1}^{2}$ & $x_{5,1,1}^{1}$ & $x_{5,5,1}^{2}$ \\
moments: & & & $x_{4,2,1}^{1}$, & $x_{4,2,1}^{2}$, & $x_{5,3,1}^{2}$ & & & & & \\
& & & $x_{4,6,1}^{2}$, & $x_{6,3,2}^{1}$ & & & & & & \\
& & & $x_{6,2,2}^{1}$ & & & & & & & \\
Values: & 10 & 11 & 12 & 13 & 14 & 15 & 16 & 17 & 18 \\
Corresponding & $S l^{10}$ & $S l^{11}$ & $S l^{12}$ & $S l^{13}$ & $S l^{14}$ & $S l^{15}$ & $S l^{16}$ & $S l^{17}$ & $S l^{18}$ & \\
states: & & & & & & & & & \\
\hline
\end{tabular}

where:

$$
\begin{aligned}
& X=\left\{X_{1}^{1}, X_{2}^{1}, X_{3}^{1}, X_{4}^{1}, X_{4}^{2}, X_{5}^{1}, X_{5}^{2}, X_{6}^{1}\right\} \\
& X_{1}^{1}=\left(x_{1,1,1}^{1}, x_{1,2,1}^{1}, x_{1,3,1}^{1}, x_{1,4,1}^{1}\right) \text { - the moments when } \\
& \text { operations executed along } p_{1}^{1}=\left(R_{1}, R_{2}, R_{3}, R_{4}\right) \text { begin }
\end{aligned}
$$$$
\begin{aligned}
X_{2}^{1}= & \left(x_{2,1,1}^{1}, x_{2,2,1}^{1}, x_{2,3,1}^{1}, x_{2,1,2}^{1}, x_{2,2,2}^{1}, x_{2,3,2}^{1}, x_{2,1,3}^{1}, x_{2,2,3}^{1},\right. \\
& \left.x_{2,3,3}^{1}\right),
\end{aligned}
$$

$$
\begin{aligned}
& X_{3}^{1}=\left(x_{3,1,1}^{1}, x_{3,2,1}^{1}, x_{3,3,1}^{1}, x_{3,4,1}^{1}, x_{3,5,1}^{1}\right), \\
& X_{4}^{1}=\left(x_{4,1,1}^{1}, x_{4,2,1}^{1}, x_{4,3,1}^{1}, x_{4,4,1}^{1}, x_{4,5,1}^{1}, x_{4,6,1}^{1}\right), \\
& X_{4}^{2}=\left(x_{4,1,1}^{2}, x_{4,2,1}^{2}, x_{4,3,1}^{2}, x_{4,4,1}^{2}, x_{4,5,1}^{2}, x_{4,6,1}^{2}\right), \\
& X_{5}^{1}=\left(x_{5,1,1}^{1}, x_{5,2,1}^{1}, x_{5,3,1}^{1}, x_{5,4,1}^{1}, x_{5,5,1}^{1}\right),
\end{aligned}
$$

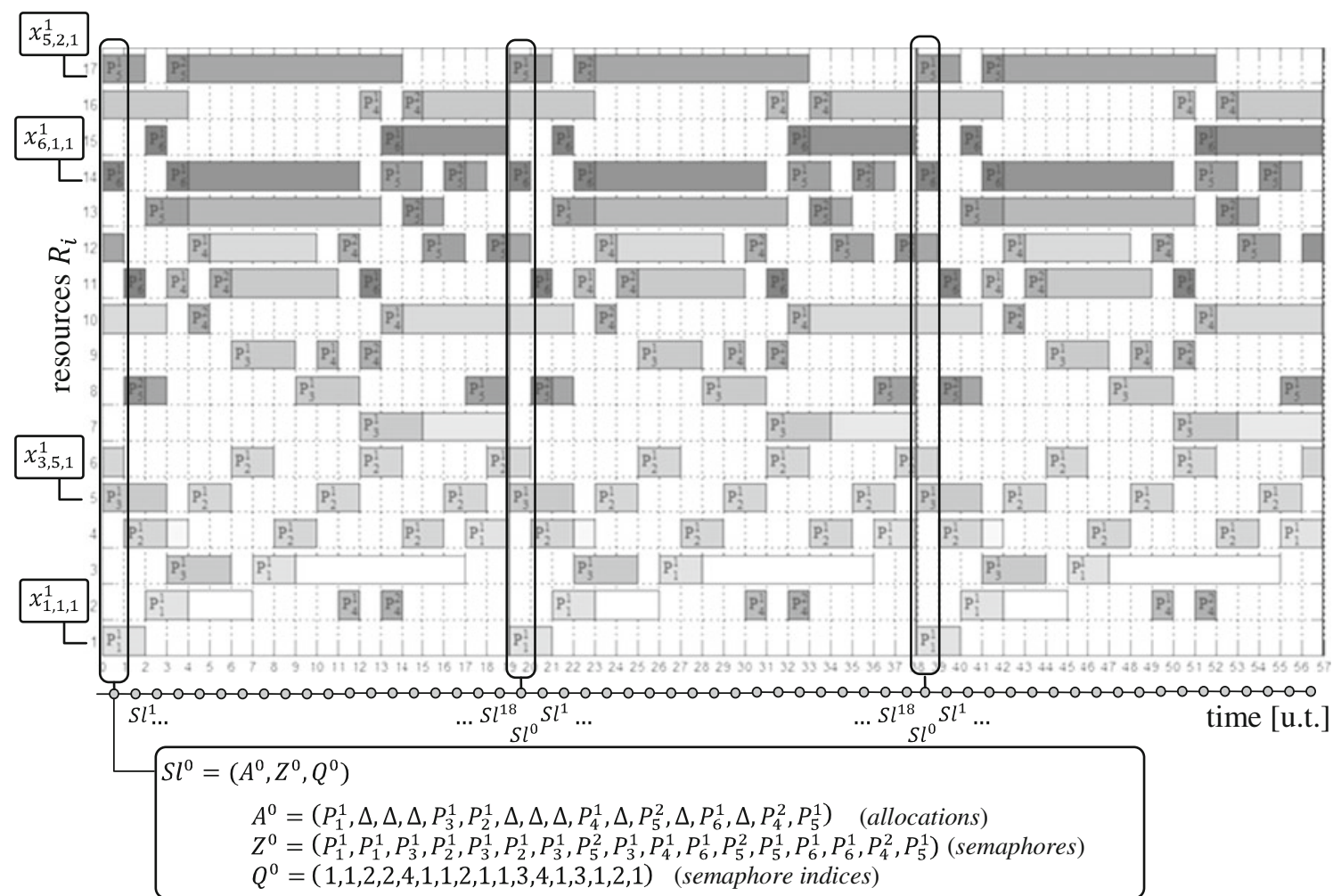

Legend:

$P_{i}^{j}$ - execution of stream $P_{i}^{j}$

$\square$ - suspension of the stream $\stackrel{S l^{i}}{\rightarrow} \rightarrow-i$-th local state of SCCP

Fig. 6 Gantt chart illustrating the cyclic steady state of local processes for the SCCP from Fig. 2, where $\Psi=(1,3,1,1,1,2)$ and $\Xi=1$ 
Table 6 Constraints determining the moments $m x_{i, j, k}$ when operations begin for the SCCP from Fig. 2

$$
\begin{aligned}
& \text { Multimodal process } m P_{1} \\
& m x_{1,1,1}=\min \left\{\left(x_{1,1,1}^{1}+a \cdot T c\right) \mid a \in \mathbb{Z}, x_{1,1,1}^{1}+a \cdot T c \geq m x_{1,7,1}+m t_{1,7,1}-T m\right\}, \\
& m x_{1,2,1}=\min \left\{\left(x_{1,2,1}^{1}+a \cdot T c\right) \mid a \in \mathbb{Z}, x_{1,2,1}^{1}+a \cdot T c \geq m x_{1,1,1}+m t_{1,1,1}\right\}, \\
& m x_{1,3,1}=\min \left\{\left(x_{1,3,1}^{1}+a \cdot T c\right) \mid a \in \mathbb{Z}, x_{1,3,1}^{1}+a \cdot T c \geq m x_{1,2,1}+m t_{1,2,1}\right\}, \\
& m x_{1,4,1}=\min \left\{\left(x_{3,2,1}^{1}+a \cdot T c\right) \mid a \in \mathbb{Z}, x_{3,2,1}^{1}+a \cdot T c \geq m x_{1,3,1}+m t_{1,3,1}\right\}, \\
& m x_{1,5,1}=\min \left\{\left(x_{3,3,1}^{1}+a \cdot T c\right) \mid a \in \mathbb{Z}, x_{3,3,1}^{1}+a \cdot T c \geq m x_{1,4,1}+m t_{1,4,1}\right\}, \\
& m x_{1,6,1}=\min \left\{\left(x_{5,2,1}^{1}+a \cdot T c\right),\left(x_{5,2,1}^{2}+a \cdot T c\right) \mid a \in \mathbb{Z}, x_{5,2,1}^{1}+a \cdot T c \geq m x_{1,5,1}+\right. \\
& \left.m t_{1,5,1}, x_{5,2,1}^{2}+a \cdot T c \geq m x_{1,5,1}+m t_{1,5,1}\right\}, \\
& m x_{1,7,1}=\min \left\{\left(x_{5,3,1}^{1}+a \cdot T c\right),\left(x_{5,3,1}^{2}+a \cdot T c\right) \mid a \in \mathbb{Z}, x_{5,2,1}^{1}+a \cdot T c\right. \\
& \left.\geq m x_{1,5,1}+m t_{1,5,1}, x_{5,2,1}^{2}+a \cdot T c \geq m x_{1,6,1}+m t_{1,6,1}\right\}
\end{aligned}
$$

Multimodal process $m P_{2}$

$$
\begin{aligned}
m x_{2,1,1}= & \min \left\{\left(x_{6,3,1}^{1}+a \cdot T c\right),\left(x_{6,3,2}^{1}+a \cdot T c\right) \mid a \in \mathbb{Z}, x_{6,3,1}^{1}+a \cdot T c \geq m x_{2,7,1}+\right. \\
& \left.m t_{2,7,1}-T m, x_{6,3,2}^{1}+a \cdot T c \geq m x_{2,7,1}+m t_{2,7,1}-T m\right\} \\
m x_{2,2,1}= & \min \left\{\left(x_{6,1,1}^{1}+a \cdot T c\right),\left(x_{6,1,2}^{1}+a \cdot T c\right) \mid a \in \mathbb{Z}, x_{6,1,1}^{1}+a \cdot T c\right. \\
\geq & \left.m x_{2,1,1}+m t_{2,1,1}, x_{6,1,2}^{1}+a \cdot T c \geq m x_{2,1,1}+m t_{2,1,1}\right\} \\
m x_{2,3,1}= & \min \left\{\left(x_{5,5,1}^{1}+a \cdot T c\right),\left(x_{5,5,1}^{2}+a \cdot T c\right) \mid a \in \mathbb{Z}, x_{5,5,1}^{1}+a \cdot T c\right. \\
\geq & \left.m x_{2,2,1}+m t_{2,2,1}, x_{5,5,1}^{2}+a \cdot T c \geq m x_{2,2,1}+m t_{2,2,1}\right\} \\
m x_{2,4,1}= & \min \left\{\left(x_{5,1,1}^{1}+a \cdot T c\right),\left(x_{5,1,1}^{2}+a \cdot T c\right) \mid a \in \mathbb{Z}, x_{5,1,1}^{1}+a \cdot T c\right. \\
\geq & \left.m x_{2,3,1}+m t_{2,3,1}, x_{5,1,1}^{2}+a \cdot T c \geq m x_{2,3,1}+m t_{2,3,1}\right\} \\
m x_{2,5,1}= & \min \left\{\left(x_{3,4,1}^{1}+a \cdot T c\right) \mid a \in \mathbb{Z}, x_{3,4,1}^{1}+a \cdot T c \geq m x_{2,4,1}+m t_{2,4,1}\right\} \\
m x_{2,6,1}= & \min \left\{\left(x_{3,5,1}^{1}+a \cdot T c\right) \mid a \in \mathbb{Z}, x_{3,5,1}^{1}+a \cdot T c \geq m x_{2,5,1}+m t_{2,5,1}\right\}, \\
m x_{2,7,1}= & \min \left\{\left(x_{2,3,1}^{1}+a \cdot T c\right),\left(x_{2,3,2}^{1}+a \cdot T c\right),\left(x_{2,3,3}^{1}+a \cdot T c\right) \mid a \in \mathbb{Z}, x_{2,3,1}^{1}+a \cdot T c \geq m x_{2,6,1}+m t_{2,6,1}, x_{2,3,2}^{1}+a \cdot T c\right. \\
& \quad \geq m x_{2,6,1}+m t_{2,6,1}, x_{2,3,3}^{1}+a \cdot T c
\end{aligned}
$$

$$
\begin{aligned}
& X_{5}^{2}=\left(x_{5,1,1}^{2}, x_{5,2,1}^{2}, x_{5,3,1}^{2}, x_{5,4,1}^{2}, x_{5,5,1}^{2}\right), \\
& X_{6}^{1}=\left(x_{6,1,1}^{1}, x_{6,2,1}^{1}, x_{6,3,1}^{1}, x_{6,1,2}^{1}, x_{6,2,2}^{1}, x_{6,3,2}^{1}\right),
\end{aligned}
$$

$C$ - the set of constraints that (due to (11)) consists of the constraints from Table 4.

The solution of the CS(14) implemented in the Oz/Mozart platform (on an Intel Core Duo $3.00 \mathrm{GHz}$

Table 7 Values of the moments $m x_{i, j, k}$ when operations begin for the SCCP from Fig. 2
Starting moments:

Values:

Corresponding local states:

Corresponding multimodal states:

Starting moments:

Values:

Corresponding local states:

Corresponding multimodal states:

\begin{tabular}{llllll}
$m x_{1,1,1}$, & $m x_{1,2,1}$ & $m x_{1,3,1}$ & $m x_{2,3,1}$ & $m x_{2,4,1}$ & $m x_{1,4,1}$ \\
$m x_{2,2,1}$ & & & & & \\
0 & 2 & 7 & 15 & 17 & 25 \\
$S l^{0}$ & $S l^{2}$ & $S l^{7}$ & $S l^{15}$ & $S l^{17}$ & $S l^{6}$ \\
$S^{0}$ & $S^{2}$ & $S x^{7}$ & $S^{15}$ & $S^{17}$ & $S^{25}$ \\
$m x_{1,5,1}$ & $m x_{2,5,1}$ & $m x_{1,6,1}$, & $m x_{1,7,1}$ & $m x_{2,7,1}$ & $m x_{2,1,1}$ \\
& & $m x_{2,6,1}$ & & & \\
28 & 31 & 38 & 40 & 44 & 51 \\
$S l^{9}$ & $S l^{12}$ & $S l^{0}$ & $S l^{2}$ & $S l^{6}$ & $S l^{17}$ \\
$S^{28}$ & $S^{31}$ & $S^{38}$ & $S^{40}$ & $S^{40}$ & $S^{51}$ \\
\hline
\end{tabular}


with 4.00-GB RAM and obtained in less than $1 \mathrm{~s}$ ) is shown in Table 5.

Therefore, the period $T c$ of the obtained cyclic steady state is equal to19. The moments when operations begin in the local processes are shown in Table 5. A Gantt chart of the cyclic steady state behavior is shown in Fig. 6. Note that each unit time corresponds to a system state, i.e., the allocation of processes to resources. Thus, the cyclic steady state consists of 19 states: $S c=\left\{S l^{0}, S l^{1}, \ldots, S l^{18}\right\}$. Similarly to Fig. 4, the states $S l$ encompassing the succeeding allocations are denoted by filled circles (see Fig. 5).

\subsection{Scheduling of multimodal processes}

Let us consider the cyclic steady state $S c$ of the local processes of an SCCP. What is the cyclic steady state of the multimodal processes $m S c$ executed in this system? Let us assume two multimodal processes $m P_{1}$ and $m P_{2}$. Therefore, we must solve the relevant CSP, i.e., $C S(S C)$ defined as
(9), which can be seen in terms of the problem (14) augmented by variables $m X=\left\{m X_{1}, m X_{2}\right\}$, where:

$$
m X_{1}=\left(m x_{1,1,1}, m x_{1,2,1}, m x_{1,3,1}, m x_{1,4,1}, m x_{1,5,1}, m x_{1,6,1}, m x_{1,7,1}\right)
$$$$
m X_{2}=\left(m x_{2,1,1}, m x_{2,2,1}, m x_{2,3,1}, m x_{2,4,1}, m x_{2,5,1}, m x_{2,6,1}, m x_{2,7,1}\right),
$$

as well as by the constraints (stated in (13)) specified in Table 6. The obtained solution consists of the solution (see Table 5) to the problem (14) that has already been obtained, as well as of the moments $m X_{1}$ and $m X_{2}$ when operations begin (see the Table 7).

The Gantt diagram of the cyclic steady state of behavior of multimodal processes (composed of 57 states $S c=$ $\left.S^{0}, S^{1}, \ldots, S^{57}\right)$ is shown in Fig. 7. The obtained period is equal to $T m=57$ (i.e., the multiple of the period $T c=19$ ). This means that within that period, the multimodal processes complete one period, whereas the local processes complete three.

The obtained cyclic steady states $S c$ and $m S c$ are shown in Fig. 8 (they are denoted as $S c_{1}$ and $m S c_{1}$, respectively). Apart from obtaining feasible solutions encompassing a cyclic behavior

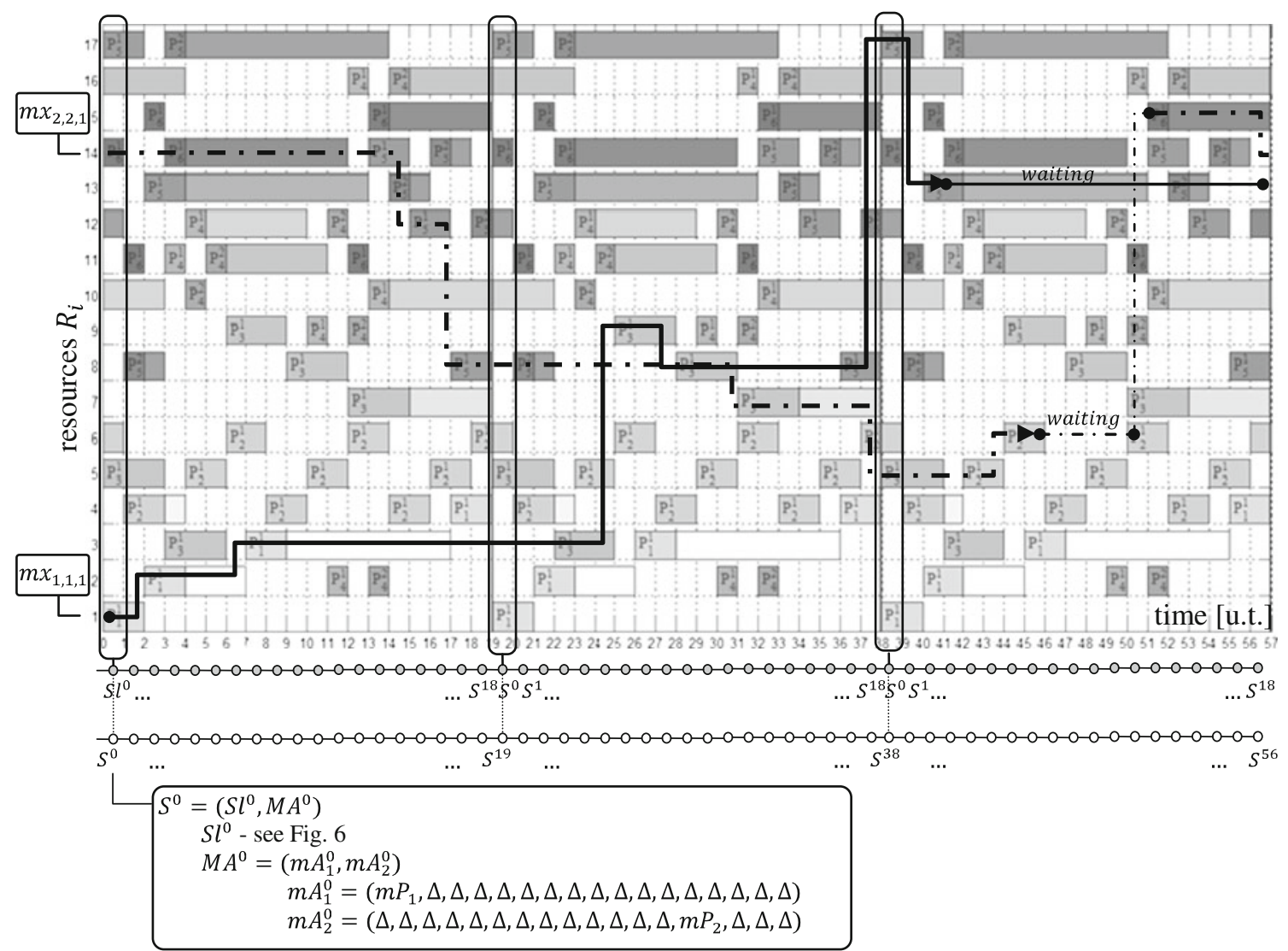

Legend:
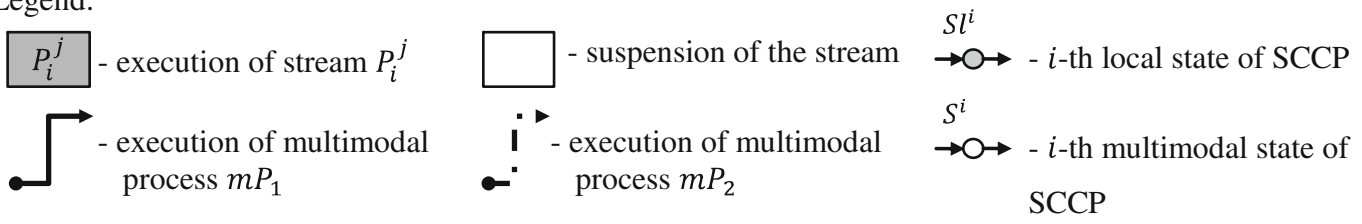

Fig. 7 Gantt chart of the cyclic steady state of multimodal processes of the SCCP from Fig. 2, where $\Psi=(1,3,1,1,1,2), \Xi=1, m \Psi(1,1)$, and $m \Xi=1$ 
Fig. 8 Space of cyclic steady states of the SCCP from Fig. 2

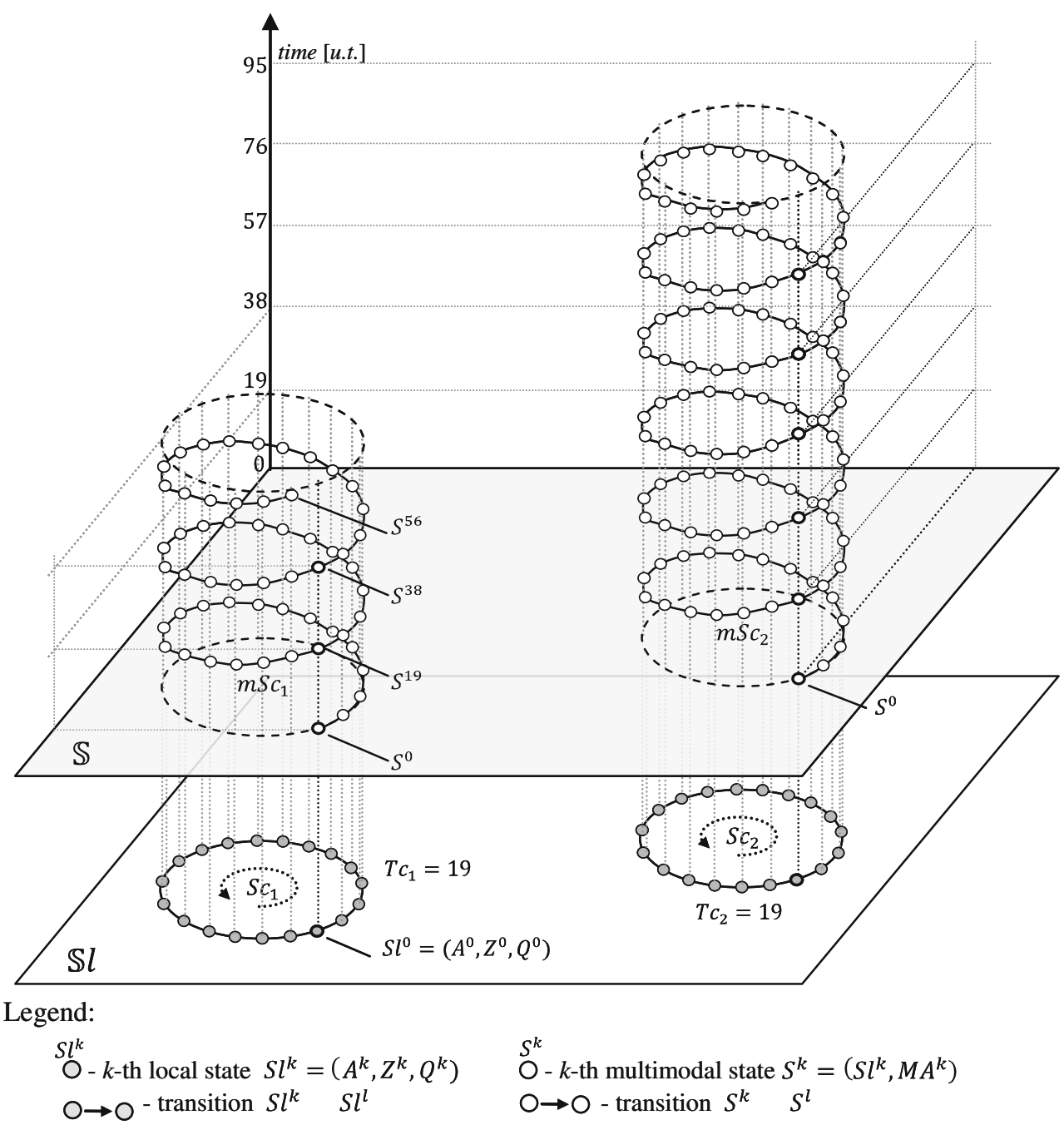

of the considered SCCP, we present another solution that follows a new dispatching rule $\sigma_{8}=\left(P_{3}^{1}, P_{5}^{1}, P_{5}^{2}\right)$ (previously, the dispatching rule associated with $R_{8}$ was $\left.\sigma_{8}=\left(P_{5}^{1}, P_{5}^{2}, P_{3}^{1}\right)\right)$. Note that a change in the dispatching rule does not change the period of local processes (even though it is different from the previous $S_{c_{2}}$ ). However, the period of the cyclic steady state of the multimodal process $m S c_{2}$ does change: $T m=113$.

Moreover, the mutual proportions changed as well: $m \Psi=$ $(3,2)$. This means that during three executions of the process $m P_{1}$, the process $m P_{2}$ executes twice. The Gantt chart of the solution of the $C S(9)$ problem (obtained in less than $1 \mathrm{~s}$ ) is shown in Fig. 9.

The provided examples demonstrate the computational efficiency of the approach based on the CSP concept (i.e., $S C(2)$ and $C S(S C)$ ) aimed at generating a cyclic steady state of the SCCP and determined by $U, T, \Theta, \Psi, \Xi, M, m T, m \Psi$, and $m \Xi$. The obtained cyclic steady state behaviors are free from transient periods, i.e., transient periods as shown in Fig. 4.
This property is crucial for problems regarding switching between possible cyclic behaviors of an SCCP [3, 4].

It should be noted, however, that for certain cases of $C S$ $(S C)$, cyclic solutions do not exist at all. This means that the constraints employed in the $C S$ can be seen merely as feasible conditions guaranteeing cyclic behavior.

In general, the lack of any cyclic behavior implies that the conditions are contradictory. However, the existence of contradicting conditions does not necessarily imply the lack of cyclic behavior. Moreover, it is crucial that the time-consuming generation of the state space that enables the refinement of cyclic steady states $[3,4]$ can be replaced by direct generation of cyclic states. From the perspective of computational complexity, this means that the exponential growth of the number of possible initial states (a possible cyclic steady state may be reachable from each of them) can be replaced by the examination of a number of dispatching rules that also grow exponentially albeit with a smaller prefactor. This is because our 

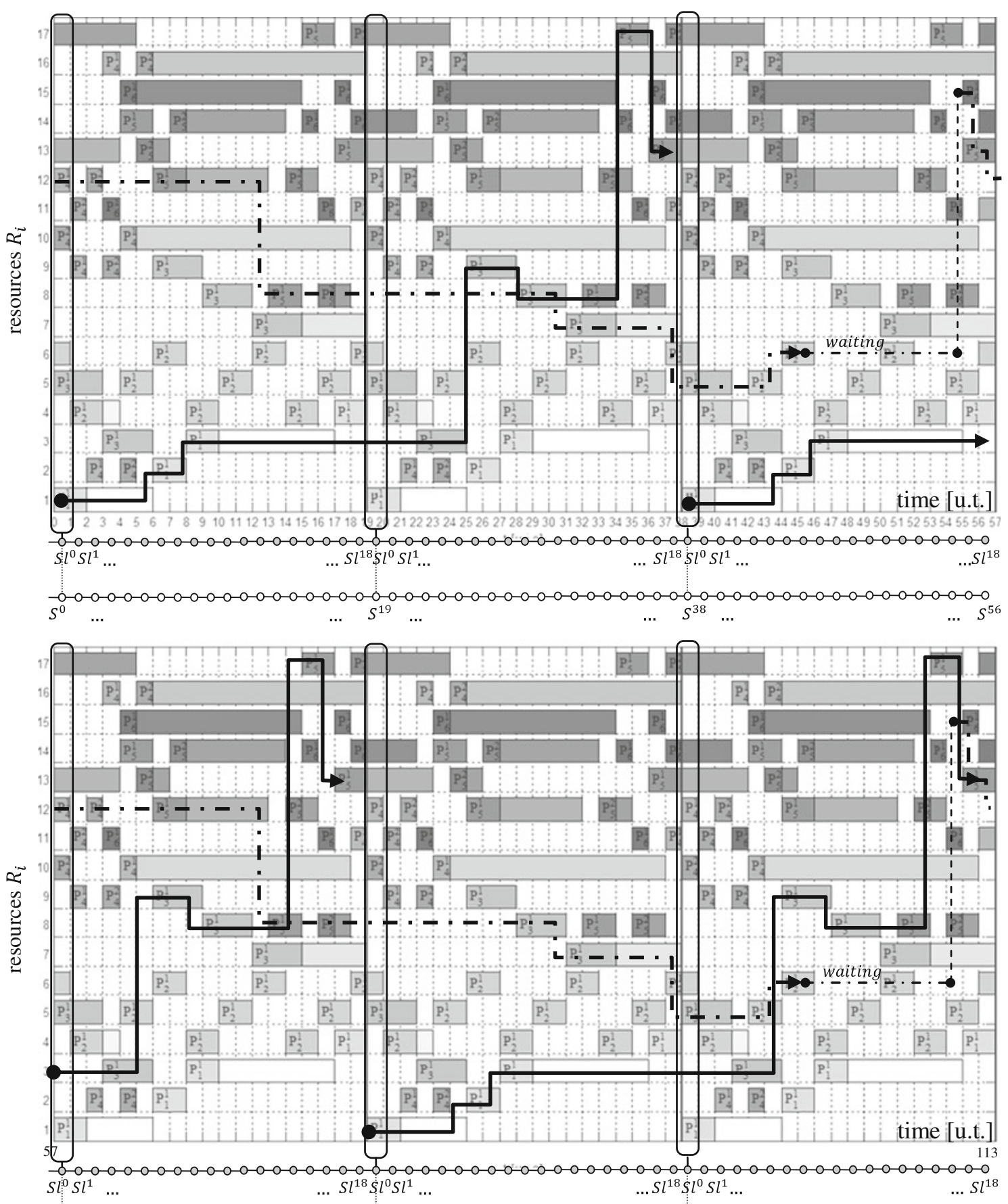

$-0-0-0-0-0-0-0-0-0-0-0-0-0-0-0-0-0-0-0-0-0-0-0-0-0-0-0-0-0-0-0-0-0-0-0-0-0-0-0-0-0-0-0-0-0-0-0-0-0-0-0-0-0-0-0-0-0$
$S^{57}$

Legend:
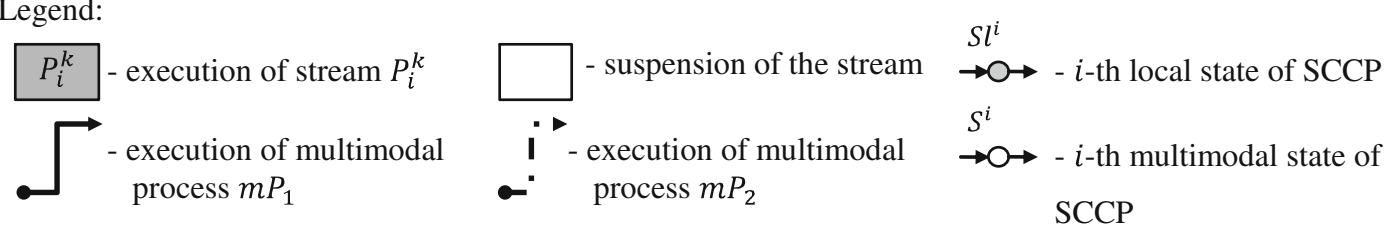

Fig. 9 The Gantt chart illustrating the cyclic behavior of the SCCP from Fig. 2, taking into account the new dispatching rule $\sigma_{8}=$ $\left(P_{3}{ }^{1}, P_{5}{ }^{1}, P_{5}{ }^{2}\right), \Psi=(1,3,1,1,1,2),=1, m \Psi(3,2)$, and $m \Xi=1$ 
time-driven framework assumes the requirements imposed on $\Xi$-periodicity of local processes (not taken into account in an event-driven framework [3, 4]), while not taking into account the information about the allocation of processes.

In general, two approaches can be distinguished. In the first one, a cyclic steady state of local processes is determined. Subsequently, basing on this, we seek the cyclic steady state of multimodal processes at the first level (see, $S M^{1}$ from Fig. 3). In turn, based on the behavior of the first level, the behavior of the second level is calculated, and so on and so forth. Such a proactive approach assumes the following statement from the formulation of the direct problem. Given a cyclic behavior at the $i$ th level, what is the cyclic behavior of multimodal processes at the $(i+1)$ th level?

Alternatively, this problem formulation can be reversed. Given a cyclic steady state of multimodal processes at the $i$ th level, what are the dispatching rules that provide the cyclic steady state of local processes guaranteeing the assumed behavior at the $i$ th level?

\section{Conclusions}

The constraint satisfaction problem of cyclic scheduling of concurrently flowing local and multimodal processes was considered. We assumed that multimodal processes are composed of sequences of local cyclic processes. For instance, if local processes encompass a network of dedicated AGV lines, the processes responsible for moving workpieces between destination points while crossing different lines constitute the multimodal processes.

Since the AGVS structure constrains its SCCP behavior, there are two fundamental problems. Does there exist such a set of dispatching rules subject to constraints on the AGVS structure that would guarantee a solution to a CSP representation of the cyclic scheduling problem? What set of dispatching rules subject to the assumed cyclic behavior of the AGVS would guarantee the solution to a CSP representation of the cyclic scheduling problem?

In this context, we propose a declarative framework providing a unified framework for the evaluation of local and multimodal processes which allows to take into account both the direct and inverse formulation of cyclic scheduling problems. Moreover, the employed framework enables evaluating the SCCP behavior on the basis of the given process layout, operation times, and the employed dispatching rules, particularly in order to compose elementary systems so as to obtain the required quantitative and qualitative behavioral features.

Therefore, our main goal is to look for a method that allows to replace time-consuming and exhaustive search for the admissible control design of an SCCP, which will be obtained by a step-by-step structural design of the SCCP that would guarantee its required behavior.
Open Access This article is distributed under the terms of the Creative Commons Attribution License which permits any use, distribution, and reproduction in any medium, provided the original author(s) and the source are credited.

\section{References}

1. Bocewicz G, Wójcik R, Banaszak Z (2009) On undecidability of cyclic scheduling problems. In: Mapping relational databases to the semantic web with original meaning, lecture notes in computer science, vol. 5914. Springer, Berlin, pp 310321

2. Bocewicz G, Bach I, Banaszak Z (2009) Logic-algebraic method based and constraints programming driven approach to AGVs scheduling. Int J Intel Inf Database Syst 3(1):56-74

3. Bocewicz G, Wójcik R, Banaszak Z (2011) Toward cycling scheduling of concurrent multimodal processes. In: Jedrzejowicz P, Nguyen NT, Hoang K (eds) Computational collective intelligence: technologies and applications, lecture notes in artificial intelligence, LNAI, vol. 6922. Springer, Berlin, pp 448-457

4. Bocewicz G, Wójcik R, Banaszak Z (2011) Cyclic steady state refinement. In: Abraham A, Corchado JM, Rodríguez González S, de Paz Santana JF (eds) International symposium on distributed computing and artificial intelligence, series: advances in intelligent and soft computing, vol. 91. Springer, Berlin, pp 191-198

5. Bocewicz G, Banaszak Z (2013) Declarative approach to cyclic scheduling of multimodal processes. In: Golińska P (ed) EcoProduction and Logistics, emerging trends and business practices, series: EcoProduction, vol. 1. Springer, Heidelberg, pp 203-238

6. Cai X, Li KN (2000) A genetic algorithm for scheduling staff of mixed skills under multi-criteria. Eur J Oper Res 125:359-369

7. Gaujal B, Jafari M, Baykal-Gursoy M, Alpan G (1995) Allocation sequences of two processes sharing a resource. IEEE Trans Robot Autom 11(5):748-353

8. Guan X, Dai X (2009) Deadlock-free multi-attribute dispatching method for $\mathrm{AGV}$ systems. Int $\mathrm{J}$ Adv Manuf Technol 45:603-615

9. Guy RK (1994) Diophantine equations. Ch. D in unsolved problems in number theory, 2nd edn. Springer, New York, pp 139-198

10. Jamili A, Ali Shafia M, Tavakkoli-Moghaddam R (2011) A hybrid algorithm based on particle swarm optimization and simulated annealing for a periodic job shop scheduling problem. Int J Adv Manuf Technol 54:309-322

11. Korytkowski P, Wisniewski T, Zaikin O (2010) Multi-criteria approach to comparison of inspection allocation for multiproduct manufacturing systems in make-to-order sector. Control Cybern 39(1):97-116

12. Lawley MA, Reveliotis SA, Ferreira PM (1998) A correct and scalable deadlock avoidance policy for flexible manufacturing systems. IEEE Trans Robot Autom 14(5):796-809

13. Levner E, Kats V, Alcaide D, Pablo L, Cheng TCE (2010) Complexity of cyclic scheduling problems: a state-of-the-art survey. Comput Ind Eng 59(2):352-361

14. Liebchen C, Möhring RH (2002) A case study in periodic timetabling. Electronic Notes Theor Comput Sci 66(6):21-34

15. Pinedo ML (2005) Planning and scheduling in manufacturing and services. Springer, New York

16. Polak M, Majdzik P, Banaszak Z, Wójcik R (2004) The performance evaluation tool for automated prototyping of concurrent cyclic processes. Fundamenta Informaticae 60(1-4):269-289

17. Schulte CH, Smolka G, Wurtz J (1998) Finite domain constraint programming in $\mathrm{Oz}$, DFKI OZ documentation series. 
German Research Center for Artificial Intelligence, Saarbrucken, Germany

18. Smart Nigiel P (1998) The algorithmic resolution of Diophantine equations. London Mathematical Society Student Text, vol 41. Cambridge University, Cambridge

19. Song J-S, Lee T-E (1998) Petri net modeling and scheduling for cyclic job shops with blocking. Comput Ind Eng 34 (2):281-295

20. Heo S-K, Lee K-H, Lee H-K, Lee I-B, Park JH (2003) A new algorithm for cyclic scheduling and design of multipurpose batch plants. Ind Eng Chem Res 42(4):836-846

21. Trouillet B, Korbaa O, Gentina J-CK (2007) Formal approach for FMS cyclic scheduling. IEEE SMC Trans, Part C 37 (1):126-137
22. Wójcik R (2007) Constraint programming approach to designing conflict-free schedules for repetitive manufacturing processes. In: Cunha PF, Maropoulos PG (eds) Digital enterprise technology. Perspectives and future challenges. Springer, New York, pp 267-274

23. Wang B, Yang H, Zhang Z-H (2007) Research on the train operation plan of the Beijing-Tianjin inter-city railway based on periodic train diagrams. Tiedao Xuebao/J China Railway Soc 29(2):8-13

24. Von Kampmeyer T (2006) Cyclic scheduling problems, Ph.D. Dissertation, Fachbereich Mathematik/Informatik, Universität Osnabrück

25. Zandong H, Lee G (2005) Application of Petri nets for deadlock analysis and avoidance in flexible manufacturing systems. Int $\mathrm{J}$ Adv Manuf Technol 25:735-742 